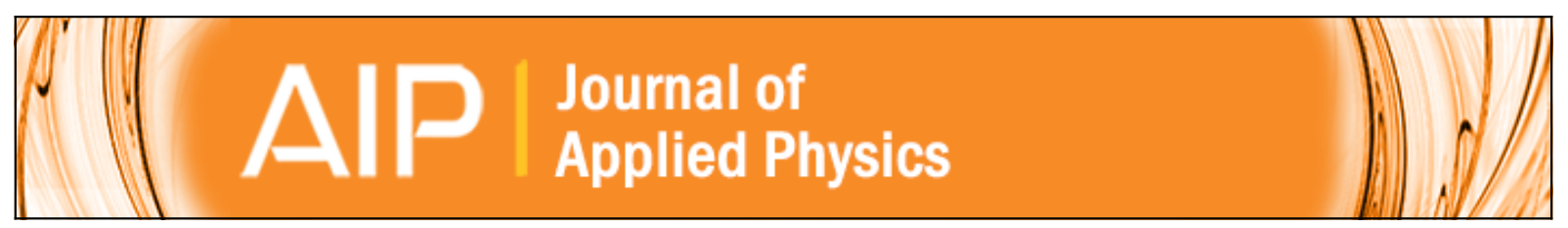

\title{
Theory of photovoltaic characteristics of semiconductor quantum dot solar cells
}

Yuchang Wu and Levon V. Asryan

Citation: Journal of Applied Physics 120, 084302 (2016); doi: 10.1063/1.4961046

View online: http://dx.doi.org/10.1063/1.4961046

View Table of Contents: http://scitation.aip.org/content/aip/journal/jap/120/8?ver=pdfcov

Published by the AIP Publishing

\section{Articles you may be interested in}

Improved conversion efficiency of InN/p-GaN heterostructure solar cells with embedded InON quantum dots Appl. Phys. Lett. 108, 061603 (2016); 10.1063/1.4941796

Material and device characteristics of InAs/GaAsSb sub-monolayer quantum dot solar cells Appl. Phys. Lett. 107, 153103 (2015); 10.1063/1.4933272

$\mathrm{Ge} / \mathrm{Si}$ quantum dots thin film solar cells

Appl. Phys. Lett. 103, 082101 (2013); 10.1063/1.4818999

Quantum-dot density dependence of power conversion efficiency of intermediate-band solar cells

J. Appl. Phys. 112, 124515 (2012); 10.1063/1.4771925

Effect of ZnS coating on the photovoltaic properties of CdSe quantum dot-sensitized solar cells J. Appl. Phys. 103, 084304 (2008); 10.1063/1.2903059

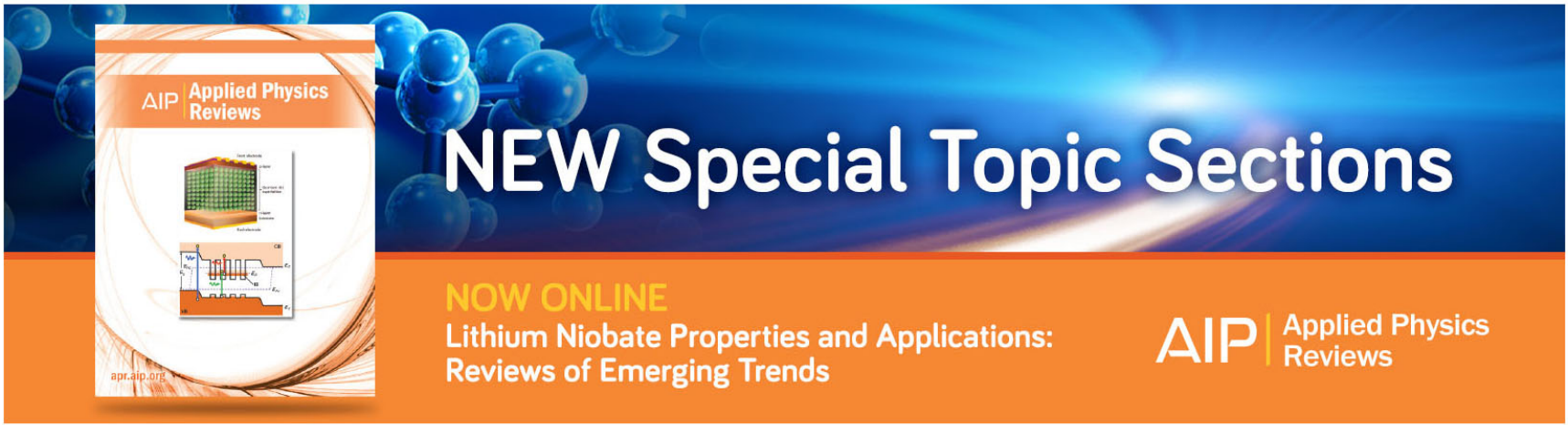




\title{
Theory of photovoltaic characteristics of semiconductor quantum dot solar cells
}

\author{
Yuchang $\mathrm{Wu}^{1,2, \mathrm{a})}$ and Levon V. Asryan ${ }^{3, \mathrm{~b})}$ \\ ${ }^{1}$ Low Carbon Energy Institute, China University of Mining and Technology, Xuzhou 221116, China \\ ${ }^{2}$ School of Materials Science and Engineering, China University of Mining and Technology, Xuzhou 221116, \\ China \\ ${ }^{3}$ Virginia Polytechnic Institute and State University, Blacksburg, Virginia 24061, USA
}

(Received 15 April 2016; accepted 3 August 2016; published online 23 August 2016)

\begin{abstract}
We develop a comprehensive rate equations model for semiconductor quantum dot solar cells (QDSCs). The model is based on the continuity equations with a proper account for quantum dots (QDs). A general analytical expression for the total current density is obtained, and the currentvoltage characteristic is studied for several specific situations. The degradation in the open circuit voltage of the QDSC is shown to be due to strong spontaneous radiative recombination in QDs. Due to small absorption coefficient of the QD ensemble, the improvement in the short circuit current density is negligible if only one QD layer is used. If spontaneous radiative recombination would be suppressed in QDs, a QDSC with multiple QD layers would have significantly higher short circuit current density and power conversion efficiency than its conventional counterpart. The effects of photoexcitation of carriers from discrete-energy states in QDs to continuum-energy states are discussed. An extended model, which includes excited states in QDs, is also introduced. Published by AIP Publishing. [http://dx.doi.org/10.1063/1.4961046]
\end{abstract}

\section{INTRODUCTION}

Due to quantum confinement of charge carriers (electrons and holes) in all three dimensions, quantum dots (QDs) have drawn much attention for their potential applications in optoelectronic devices such as solar cells (SCs). ${ }^{1-13}$ Changing the sizes of QDs and, hence, the position of confined carrier energy levels can easily alter the optical properties of QDbased devices. When QDs are used in a multi-junction SC, the current due to photon absorption by QDs can be tuned to achieve the current-match condition leading to an increase in the power conversion efficiency. ${ }^{6}$ QDs were also proposed to provide an intermediate band (IB) in an intermediate band solar cell (IBSC). ${ }^{1}$

It was shown earlier that the power conversion efficiency of an ideal IBSC $^{14,15}$ could exceed the ShockleyQueisser (SQ) limit of a single bandgap SC. ${ }^{16}$ The idea is that, besides the optical transition from the valence band (VB) to the conduction band (CB), by introducing an IB into the bandgap of the host material, an additional IB-mediated two-step two-photon-absorption process (VB $\rightarrow$ IB and the subsequent $\mathrm{IB} \rightarrow \mathrm{CB}$ transitions) becomes possible. The otherwise wasted sub-bandgap energy photons can now be absorbed via IB and, hence, both the short circuit current and the overall power conversion efficiency should be increased.

In a semiconductor quantum dot solar cell (QDSC), in which one or multiple layers with QDs are embedded in a conventional semiconductor SC, the QD confined energy level $^{7}$ or the miniband ${ }^{1}$ can act as an IB. The miniband is formed when multiple layers with QDs are grown within a larger bandgap material matrix and the distance between

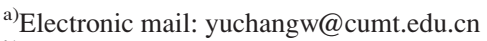

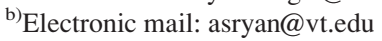

neighboring layers or the height of the potential energy barrier is small enough for tunneling to occur between the layers. Making high performance QDSC turned, however, to be a difficult task. The problem is that, although the short circuit current can be increased due to absorption of subbandgap energy photons, the open circuit voltage is decreased. The increase in the short circuit current can hardly compensate for the decrease in open circuit voltage and the overall effect is a reduction of the power conversion efficiency.

Due to the fact that zero-dimensional QDs are incorporated in the bulk material and due to the presence of multiple processes in QDSCs, certain simplifications were made to provide theoretical insights into the operating characteristics of QDSCs. ${ }^{17-22}$ For instance, the confined electron energy level in a QD or the miniband were treated as the IB. The VB offset was assumed to be zero. While the absorption coefficient of a QD ensemble is energy-dependent (with a peak at the photon energy corresponding to the optical transition in a mean-sized QD), the IB was characterized by two constant absorption coefficients-one for a range of subbandgap energy photons corresponding to the VB $\rightarrow$ IB transitions and the other for the IB $\rightarrow \mathrm{CB}$ optical transitions. QDs were also assumed to be evenly distributed in the active region. The carrier dynamics between QDs and the host material and the spontaneous radiative recombination within QDs were also not properly included into the existing models. Most of the present QDSC theories consider InAs/GaAs QDSCs, in which InAs QDs are grown in the GaAs matrix and which are relatively easy to fabricate. The problem is, however, that the energy levels of carriers confined in QDs correspond to the GaAs bandgap. The VB and CB offset energies are not zero, they are actually large, which is far 
from an ideal situation. ${ }^{9}$ As a result, transitions between the electron- and hole-ground states in QDs and carrier exchange (via both thermal and non-thermal processes) between the confined states in QDs and the continuous energy states in the bulk will occur. Hence, two constant absorption coefficients cannot properly describe the presence of QDs in a QDSC.

In this paper, we develop a rate equations model for semiconductor QDSCs based on such a model for QD lasers. ${ }^{23,24}$ Unlike lasers, which operate by current injection, a QDSC is a power source and hence the photocarrier generation term should now enter into the rate equations. We use the Dirac delta function to describe spatial discreteness of a QD layer. ${ }^{24}$ The carrier thermal exchange between QDs and the host material is characterized by capture cross-sections. Photon absorptions both via the QD ground-state transitions and photoexcitation are studied. We show that inferior performance of QDSCs as compared to their conventional counterpart is due to weak photon absorption in QDs and weak photoexcitation of carriers from QDs as well as to strong spontaneous radiative recombination in QDs.

\section{THEORETICAL MODEL}

Our model is based on the following set of steadystate rate equations that describe the carrier generation, recombination, and transport processes in a QDSC as shown in Fig. 1:

For free carriers in the bulk i-region

$$
\begin{gathered}
\sigma_{\mathrm{n}} \mathrm{v}_{\mathrm{n}} n_{1} N_{\mathrm{S}} f_{\mathrm{n}} \delta\left(x-x_{0}\right)-\sigma_{\mathrm{n}} \mathrm{v}_{\mathrm{n}} N_{\mathrm{S}}\left(1-f_{\mathrm{n}}\right) n(x) \delta\left(x-x_{0}\right) \\
+g_{\text {non_th, } \mathrm{n}} \delta\left(x-x_{0}\right)+g_{\text {bulk }}-R_{\text {bulk }}+\frac{1}{q} \frac{d j_{\mathrm{n}}}{d x}=0 \\
\sigma_{\mathrm{p}} \mathrm{v}_{\mathrm{p}} p_{1} N_{\mathrm{S}} f_{\mathrm{p}} \delta\left(x-x_{0}\right)-\sigma_{\mathrm{p}} \mathrm{v}_{\mathrm{p}} N_{\mathrm{S}}\left(1-f_{\mathrm{p}}\right) p(x) \delta\left(x-x_{0}\right) \\
+g_{\text {non_th,p }} \delta\left(x-x_{0}\right)+g_{\text {bulk }}-R_{\text {bulk }}-\frac{1}{q} \frac{d j_{\mathrm{p}}}{d x}=0
\end{gathered}
$$

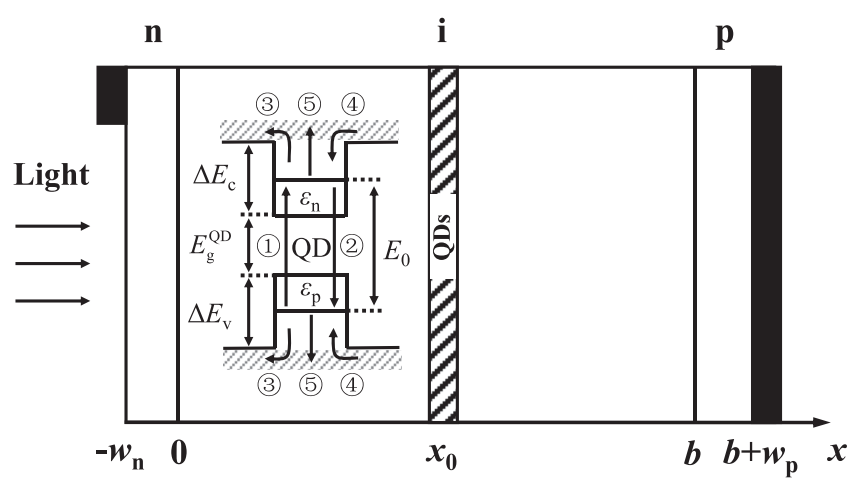

FIG. 1. Simplified structure and schematic energy band diagram (the inset) of a typical QDSC (not drawn to scale). The layer with QDs is located at the position $x_{0}$ in the i-region. The thickness of the n-, i-, and p-region is $w_{\mathrm{n}}, b$, and $w_{\mathrm{p}}$, respectively. The front and back contacts are shown in black. The main processes (shown by arrows) associated with QDs are as follows: (1) photocarrier generation by absorption of photons, (2) radiative recombination of carriers, (3) thermal escape of carriers from QDs to the bulk, (4) carrier capture from the bulk into QDs, and (5) photoexcitation or tunneling of carriers confined in QDs. For our calculations, a typical InAs/GaAs QDSC is used; $w_{\mathrm{n}}=300 \mathrm{~nm}, b=1000 \mathrm{~nm}, w_{\mathrm{p}}=200 \mathrm{~nm}, x_{0}=b / 2$, and the surface recombination velocities for the front and back contacts $S_{\mathrm{p}}=S_{\mathrm{n}}=10^{4} \mathrm{~cm} / \mathrm{s}$. The doping levels in the $\mathrm{n}$ - and p-region are $2 \times 10^{16}$ and $4 \times 10^{17} \mathrm{~cm}^{-3}$, respectively. for carriers confined in QDs

$$
\begin{aligned}
& \sigma_{\mathrm{n}} \mathrm{v}_{\mathrm{n}} N_{\mathrm{S}}\left(1-f_{\mathrm{n}}\right) n(x) \delta\left(x-x_{0}\right)-\sigma_{\mathrm{n}} \mathrm{v}_{\mathrm{n}} n_{1} N_{\mathrm{S}} f_{\mathrm{n}} \delta\left(x-x_{0}\right) \\
& \quad-g_{\text {non_th, } \mathrm{n}} \delta\left(x-x_{0}\right)+g_{\mathrm{QD}} \delta\left(x-x_{0}\right)-R_{\mathrm{QD}} \delta\left(x-x_{0}\right)=0,
\end{aligned}
$$

$$
\begin{aligned}
& \sigma_{\mathrm{p}} \mathrm{v}_{\mathrm{p}} N_{\mathrm{S}}\left(1-f_{\mathrm{p}}\right) p(x) \delta\left(x-x_{0}\right)-\sigma_{\mathrm{p}} \mathrm{v}_{\mathrm{p}} p_{1} N_{\mathrm{S}} f_{\mathrm{p}} \delta\left(x-x_{0}\right) \\
& \quad-g_{\text {non_th,p }} \delta\left(x-x_{0}\right)+g_{\mathrm{QD}} \delta\left(x-x_{0}\right)-R_{\mathrm{QD}} \delta\left(x-x_{0}\right)=0,
\end{aligned}
$$

where $n(x)$ and $p(x)$ are the free electron and hole densities in the i-region, respectively, $\sigma_{\mathrm{n}, \mathrm{p}}$ are the cross-sections of carrier capture into a $\mathrm{QD}, \mathrm{v}_{\mathrm{n}, \mathrm{p}}$ are the carrier thermal velocities in the bulk material, $N_{\mathrm{S}}$ is the surface density of QDs (i.e., the number of QDs per unit area of the QD layer), the quantities $n_{1}=N_{\mathrm{c}} \exp \left[-\left(\Delta E_{\mathrm{c}}-\varepsilon_{\mathrm{n}}\right) / T\right]$ and $p_{1}=N_{\mathrm{v}} \exp \left[-\left(\Delta E_{\mathrm{v}}-\varepsilon_{\mathrm{p}}\right) / T\right]$ characterize the carrier thermal escape from a QD to the bulk, $N_{\mathrm{c}, \mathrm{v}}$ are the effective densities of states in the bulk, $\Delta E_{\mathrm{c}, \mathrm{v}}$ are the energy band offsets, $\varepsilon_{\mathrm{n}, \mathrm{p}}$ are the confined energies, $T$ is the temperature (in units of energy), $f_{n, p}=\{1$ $\left.+\exp \left[\left(\varepsilon_{\mathrm{n}, \mathrm{p}}-\mu_{\mathrm{n}, \mathrm{p}}\right) / T\right]\right\}^{-1}$ are the confined carrier level occupancies in QDs, and $\mu_{\mathrm{n}, \mathrm{p}}$ are the quasi-Fermi levels. Both $\mu_{\mathrm{n}, \mathrm{p}}$ and $\varepsilon_{\mathrm{n}, \mathrm{p}}$ are measured from the respective band edges in the QD material (see the inset in Fig. 1 for $\varepsilon_{\mathrm{n}, \mathrm{p}}$; the positions of $\mu_{\mathrm{n}, \mathrm{p}}$ will be determined later). $g_{\text {bulk }}$ and $R_{\text {bulk }}$ are the photocarrier generation and radiative recombination rates in the i-region, respectively. $q$ is the elementary charge, $j_{\mathrm{n}}$ and $j_{\mathrm{p}}$ are the electron and hole current densities in the i-region, respectively.

Carriers confined in QDs are involved in the following processes: generation due to absorption of photons with a rate $g_{\mathrm{QD}}$ (process (1) in Fig. 1), radiative recombination with a rate $R_{\mathrm{QD}}$ (process (2) in Fig. 1), thermal escape to the bulk (process (3) in Fig. 1) or non-thermal escape to the bulk (photoexcitation or tunneling; process (5) in Fig. 1). The first terms in the left-hand side of Eqs. (1) and (2) are the rates of electron and hole thermal escape from QDs to the bulk, respectively. $g_{\text {non_th,n,p }}$ are the rates of non-thermal escape. Once electrons (holes) escape to the bulk, they can drift across the i-region to the $n-(p-)$ region due to the built-in electric field. Free electrons and holes can also be captured back from the bulk into QDs (process (4) in Fig. 1); this process is described by the second terms in the left-hand side of Eqs. (1) and (2).

The QD layer occupies only a very small fraction of the i-region, and hence, we use the Dirac delta function $\delta\left(x-x_{0}\right)$ (where $x_{0}$ is the location of the QD layer) in the rate equations to describe the QD-related local events.

\section{RESULTS AND DISCUSSIONS}

\section{A. General expression for the total current density}

The free carrier densities $n(x)$ and $p(x)$ can be discontinuous at the QD layer $x=x_{0}$. Defining the integration of the product of the Dirac delta-function and a discontinuous function as follows: 


$$
\begin{aligned}
& \int_{x_{0}-0}^{x_{0}+0} \delta\left(x-x_{0}\right) n(x) d x \\
& \quad=\lim _{\Delta \rightarrow 0}\left\{\frac{1}{\Delta \sqrt{2 \pi}} \int_{x_{0}-0}^{x_{0}+0} \exp \left[-\frac{\left(x-x_{0}\right)^{2}}{2 \Delta^{2}}\right] n(x) d x\right\},
\end{aligned}
$$

and integrating (3) and (4) over an infinitesimal interval around $x=x_{0}$, we obtain

$$
\begin{aligned}
& \sigma_{\mathrm{n}} \mathrm{v}_{\mathrm{n}} N_{\mathrm{S}}\left(1-f_{\mathrm{n}}\right) \frac{n\left(x_{0}-0\right)+n\left(x_{0}+0\right)}{2} \\
& -\sigma_{\mathrm{n}} \mathrm{v}_{\mathrm{n}} n_{1} N_{\mathrm{S}} f_{\mathrm{n}}-g_{\text {non_th, } \mathrm{n}}+g_{\mathrm{QD}}-R_{\mathrm{QD}}=0, \\
& \sigma_{\mathrm{p}} \mathrm{v}_{\mathrm{p}} N_{\mathrm{S}}\left(1-f_{\mathrm{p}}\right) \frac{p\left(x_{0}-0\right)+p\left(x_{0}+0\right)}{2} \\
& \quad-\sigma_{\mathrm{p}} \mathrm{v}_{\mathrm{p}} p_{1} N_{\mathrm{S}} f_{\mathrm{p}}-g_{\text {non_th, } \mathrm{p}}+g_{\mathrm{QD}}-R_{\mathrm{QD}}=0 .
\end{aligned}
$$

From (6) and (7), we have for the average values of the free electron and hole densities at the QD layer

$$
\begin{aligned}
& \frac{n\left(x_{0}-0\right)+n\left(x_{0}+0\right)}{2}=n_{1} \frac{f_{\mathrm{n}}}{1-f_{\mathrm{n}}}+\frac{1}{\sigma_{\mathrm{n}} \mathrm{v}_{\mathrm{n}} N_{\mathrm{S}}\left(1-f_{\mathrm{n}}\right)} \\
& \times\left(R_{\mathrm{QD}}-g_{\mathrm{QD}}+g_{\text {non_th }, \mathrm{n}}\right) \text {, } \\
& \frac{p\left(x_{0}-0\right)+p\left(x_{0}+0\right)}{2} \\
& =p_{1} \frac{f_{\mathrm{p}}}{1-f_{\mathrm{p}}}+\frac{1}{\sigma_{\mathrm{p}} \mathrm{v}_{\mathrm{p}} N_{\mathrm{S}}\left(1-f_{\mathrm{p}}\right)}\left(R_{\mathrm{QD}}-g_{\mathrm{QD}}+g_{\text {non_th,p }}\right) \text {. }
\end{aligned}
$$

Instead of (1) and (2), equations

$$
g_{\text {bulk }}-R_{\text {bulk }}+\frac{1}{q} \frac{d j_{\mathrm{n}}}{d x}=0
$$

and

$$
g_{\text {bulk }}-R_{\text {bulk }}-\frac{1}{q} \frac{d j_{\mathrm{p}}}{d x}=0
$$

can be equivalently used separately for the left- and righthand sides (with respect to the QD layer) of the i-region (i.e., the regions $0 \leq x \leq x_{0}-0$ and $x_{0}+0 \leq x \leq b$, respectively) provided Eqs. (10) and (11) are supplemented with the following boundary conditions at the QD layer:

$$
\begin{aligned}
& \left.j_{\mathrm{n}}\right|_{x=x_{0}+0}-\left.j_{\mathrm{n}}\right|_{x=x_{0}-0}=q\left(R_{\mathrm{QD}}-g_{\mathrm{QD}}\right), \\
& \left.j_{\mathrm{p}}\right|_{x=x_{0}+0}-\left.j_{\mathrm{p}}\right|_{x=x_{0}-0}=q\left(g_{\mathrm{QD}}-R_{\mathrm{QD}}\right) .
\end{aligned}
$$

These boundary conditions are obtained by integrating Eqs. (1)-(4) over an infinitesimal interval around $x_{0}$.

It should be noted that, although the total current density $j=j_{\mathrm{n}}(x)+j_{\mathrm{p}}(x)$ is constant in position, the electron and hole current densities may be discontinuous at the QD layer. As can be seen from Eqs. (12) and (13), the discontinuity is due to the fact that the generation and spontaneous radiative recombination rates in QDs may not equal to each other. This discontinuity is a feature related to the use of the $\delta$-function to describe the QD layer in our model.
As seen from Eqs. (6) and (7), the net escape rate from QDs to the i-region is the same for electrons and holes [and equals to $\left.\left(g_{\mathrm{QD}}-R_{\mathrm{QD}}\right)\right]$ even if their individual capture and escape rates are different.

At the front and back contacts, the current is completely due to the majority carriers so that

$$
j=j_{\mathrm{n}}\left(-w_{\mathrm{n}}\right)=j_{\mathrm{p}}\left(b+w_{\mathrm{p}}\right) .
$$

It follows from (14) that

$$
j_{\mathrm{p}}\left(-w_{\mathrm{n}}\right)=j_{\mathrm{n}}\left(b+w_{\mathrm{p}}\right)=0 .
$$

Integrating Eq. (10) from $-w_{\mathrm{n}}$ to $b+w_{\mathrm{p}}$ and using Eq. (12), we obtain a general expression for the total current density in a QDSC

$$
\begin{aligned}
j= & q \int_{-w_{\mathrm{n}}}^{x_{0}-0}\left(g_{\text {bulk }}-R_{\text {bulk }}\right) d x \\
& +q \int_{x_{0}+0}^{b+w_{\mathrm{p}}}\left(g_{\text {bulk }}-R_{\text {bulk }}\right) d x+q\left(g_{\mathrm{QD}}-R_{\mathrm{QD}}\right) .
\end{aligned}
$$

Eq. (16) simply states that the total current density is due to the net generation of photocarriers by the entire device.

We did not consider here the nonradiative recombination loss of carriers. However, this process can be easily incorporated into our model by simply rewriting $R_{\text {bulk }}$ and $R_{\mathrm{QD}}$ to include both radiative and nonradiative recombinations in Eq. (16).

A straightforward approach to obtain the $j$ - $V$ characteristic of the QDSC would be to solve the carrier transport equations and Poisson's equation supplemented by the corresponding boundary conditions. Having thus obtained $n(x)$ and $p(x)$ and the spatial distribution of the electric field, the total current density could be calculated. In the i-region, however, we would have a set of coupled integro-differential equations [Eqs. (10) and (11)]. At the QD layer, there would be a step in the electric field equal to $N_{\mathrm{S}}\left(f_{\mathrm{p}}-f_{\mathrm{n}}\right) / \varepsilon_{0} \varepsilon_{\mathrm{r}}$, where $\varepsilon_{0}$ is the permittivity of free space and $\varepsilon_{\mathrm{r}}$ is the relative permittivity (dielectric constant). This set of integro-differential equations could only be solved numerically. Our goal here is, however, to derive analytical expressions for the $j-V$ characteristic. To do this, we use the approach which is described in Ref. 25.

Since the total current density is constant in position $\left[j=j_{\mathrm{p}}(x)+j_{\mathrm{n}}(x)=\operatorname{const}(x)\right]$, it can be calculated by adding the electron and hole current densities at any position $x$. Let us choose $x=0$ for doing this. At $x=0, j_{\mathrm{p}}(0)$ can be easily obtained by solving the ambipolar transport equation for minority holes, and $j_{\mathrm{n}}(0)$ can be obtained by integrating Eq. (10) from 0 to $b$ at which position electrons can be treated as minority carrier. With Eq. (12), the total current density is given by

$$
\begin{aligned}
j= & j_{\mathrm{p}}(0)+q \int_{0}^{x_{0}-0}\left(g_{\text {bulk }}-R_{\text {bulk }}\right) d x+q\left(g_{\mathrm{QD}}-R_{\mathrm{QD}}\right) \\
& +q \int_{x_{0}+0}^{b}\left(g_{\text {bulk }}-R_{\text {bulk }}\right) d x+j_{\mathrm{n}}(b) .
\end{aligned}
$$


In (17), the photocarrier generation rate for the bulk i-region is given by

$$
g_{\text {bulk }}(x)=\int_{0}^{\lambda_{\text {bulk }}} F \alpha_{\text {bulk }} \exp \left[-\alpha_{\text {bulk }}\left(x+w_{\mathrm{n}}\right)\right] d \lambda,
$$

where $\lambda_{\text {bulk }}$ is the longest photon wavelength for optical transitions in the bulk. The absorption coefficient $\alpha_{\text {bulk }}(\lambda)$ for the i-region material is a function of the wavelength $\lambda ;^{26}$ the photon flux $F(\lambda)$ of the incident sunlight at a certain wavelength $\lambda$ is given by

$$
F(\lambda)=2 \pi c\left(\frac{r_{\text {sun }}}{d_{\text {se }}}\right)^{2} \frac{1}{\lambda^{4}} \frac{1}{\exp \left(h c / \lambda T_{\text {sun }}\right)-1},
$$

where $c$ is the speed of light in vacuum, $r_{\text {sun }}$ is the radius of the sun, $d_{\mathrm{se}}$ is the mean distance between the sun and earth, $h$ is Planck's constant, $T_{\text {sun }}$ is set to be $5762 \mathrm{~K}^{27}$

So far, one layer with QDs was only considered. If multiple layers are used (with $Z_{\mathrm{L}}$ being their number), we will have $Z_{\mathrm{L}}$ pairs of rate equations for confined carriers. Integrating Eq. (10), we will obtain for the total current density

$$
\begin{aligned}
j= & j_{\mathrm{p}}(0)+q \int\left(g_{\text {bulk }}-R_{\text {bulk }}\right) d x \\
& +q \sum_{\mathrm{i}=1}^{Z_{\mathrm{L}}}\left(g_{\mathrm{QD}, \mathrm{i}}-R_{\mathrm{QD}, \mathrm{i}}\right)+j_{\mathrm{n}}(b),
\end{aligned}
$$

where the integration is taken over the interval $[0, b]$ with an effective thickness that does not include the total thickness of QD layers.

When multiple layers with QDs are used, tunneling can occur between neighboring QD layers (do not confuse with the tunneling escape of confined carriers to the i-region), which is ignored in Eq. (20). It has been experimentally demonstrated that such tunneling is not required for better performance. ${ }^{7}$ If this process is, however, present, the rate equations for confined carriers in each QD layer should be revised to include the in- and out-tunneling rates of carriers. As a consequence, the boundary conditions at each QD layer should also contain the in- and out-tunneling terms. Following a procedure similar to that for deriving Eq. (17), we can obtain the expression for the total current density, which has exactly the same form as Eq. (20). This is reasonable since the total current density should be given by the net generation of photocarriers. See Appendix A for the case of tunneling between neighboring QD layers.

It should be noted that Eqs. (16) and (20) for the total current density are general. They are derived from the continuity equations with the boundary conditions at the QD layer.

\section{B. Assumptions and results}

To simplify the calculations, $g_{\text {bulk }}$ can be approximated by an exponential function ${ }^{3,28}$

$$
g_{\text {bulk }}(x)=\alpha_{\text {bulk }} \phi_{0} \exp \left[-\alpha_{\text {bulk }}\left(x+w_{\mathrm{n}}\right)\right],
$$

where $\alpha_{\text {bulk }}$ and $\phi_{0}$ are numerical constants.
To obtain an analytical expression for $j$, we make the following assumptions, which are often used in the analysis of $\mathrm{p}-\mathrm{n}$ junctions: ${ }^{28}$

(i) In the n- (p-) type region (i.e., in the regions $-w_{\mathrm{n}} \leq x \leq 0$ and $b \leq x \leq b+w_{\mathrm{p}}$, respectively), holes (electrons) are minority carriers even under illumination.

(ii) n- and p-type regions are quasi-neutral so that the electric fields are zero there.

(iii) The front and back contacts can be characterized by the surface recombination velocities $S_{\mathrm{p}}$ and $S_{\mathrm{n}}$, respectively.

(iv) The space charge regions are neglected.

Using above assumptions and Eq. (21), analytical expressions for $j_{\mathrm{p}}(0)$ and $j_{\mathrm{n}}(b)$ can be easily obtained by solving the ambipolar transport equations with the following boundary conditions:

For minority holes

$$
\begin{gathered}
p(0)=p_{\text {no }} \exp (V / T), \\
\left.\frac{d p}{d x}\right|_{x=-w_{\mathrm{n}}}=\frac{S_{\mathrm{p}}}{D_{\mathrm{p}}}\left[p\left(-w_{\mathrm{n}}\right)-p_{\mathrm{no}}\right],
\end{gathered}
$$

and for minority electrons

$$
\begin{gathered}
n(b)=n_{\mathrm{po}} \exp (V / T), \\
\left.\frac{d n}{d x}\right|_{x=b+w_{\mathrm{p}}}=-\frac{S_{\mathrm{n}}}{D_{\mathrm{n}}}\left[n\left(b+w_{\mathrm{p}}\right)-n_{\mathrm{po}}\right],
\end{gathered}
$$

where $V$ is the output voltage (in units of energy), and $p_{\text {no }}$ and $n_{\text {po }}$ are the hole and electron densities at thermal equilibrium in $\mathrm{n}$ - and p-regions, respectively. $D_{\mathrm{n}, \mathrm{p}}$ are the diffusion coefficients for electrons and holes, respectively.

In the bulk i-region, the radiative recombination process is bimolecular and the electric field is present; therefore, it is impossible to calculate $R_{\text {bulk }}$ analytically. Besides, the electric field may not be constant-it is determined not only by carrier densities and doping levels but also by the net charge in the QD layer. ${ }^{29}$ Therefore, to obtain an analytical expression for the current density due to radiative recombination $j_{\text {rec }}^{\text {bulk }}$, we need to use one more assumption: ${ }^{30}$

(v) In the i-region, the drops of the quasi-Fermi levels $E_{\mathrm{Fn}, \mathrm{p}}$ for electrons and holes are so small that we approximately have

$$
E_{\mathrm{Fn}}-E_{\mathrm{Fp}}=V .
$$

Eq. (24) is equivalent to the assumption that electrons and holes have high mobilities so that they quickly drift to the $\mathrm{n}$ - and p-regions, respectively. ${ }^{19}$

With (24), we have for the recombination current density in i-region

$$
j_{\text {rec }}^{\text {bulk }}=q \int_{0}^{b} R_{\text {bulk }} d x=q b B n_{i}^{2}\left[\exp \left(\frac{V}{T}\right)-1\right],
$$

where $B$ is the radiative recombination constant for the bulk.

The current density associated with absorption of photons via ground-state transitions in QDs is given by 


$$
j_{\mathrm{ph}}^{\mathrm{QD}}=q g_{\mathrm{QD}}=j_{\mathrm{ph}}^{\mathrm{QD} \_\mathrm{max}}\left(1-f_{\mathrm{n}}-f_{\mathrm{p}}\right),
$$

where the photocarrier generation rate by the QD ensemble is

$$
g_{\mathrm{QD}}=\left|\int_{\lambda_{\text {bulk }}}^{+\infty} \sigma_{\mathrm{QD}} N_{\mathrm{S}} F d \lambda\right|\left(1-f_{\mathrm{n}}-f_{\mathrm{p}}\right) .
$$

The maximum value of $j_{\mathrm{ph}}^{\mathrm{QD}}$ (obtained when $f_{\mathrm{n}}=f_{\mathrm{p}}=0$ ) is

$$
j_{\mathrm{ph}}^{\mathrm{QD} \_m a x}=q\left|\int_{\lambda_{\text {bulk }}}^{+\infty} \sigma_{\mathrm{QD}} N_{\mathrm{S}} F d \lambda\right|
$$

In (28), $\sigma_{\mathrm{QD}}(\lambda)=\bar{a}^{3} \alpha_{\mathrm{QD}}(\lambda)$ is the cross-section (in units of $\mathrm{cm}^{2}$ ) of photon absorption by a QD, $\bar{a}$ is the mean size of QDs, and $\alpha_{\mathrm{QD}}(\lambda)$ is the absorption coefficient per QD (in units of $\mathrm{cm}^{-1}$ ) averaged over the inhomogeneously broadened ensemble of QDs ${ }^{31,32}$

$$
\alpha_{\mathrm{QD}}(\lambda)=\frac{8}{3} \pi^{2} \frac{\alpha_{\text {fine }}}{\sqrt{\varepsilon_{\mathrm{r}}}} P^{2} \frac{1}{E(\lambda)} I_{\text {overlap }} d\left\langle\frac{1}{V_{\mathrm{QD}}} \delta\left[E(\lambda)-E_{0}\right]\right\rangle,
$$

where $\alpha_{\text {fine }}$ is the fine-structure constant, $V_{\mathrm{QD}}$ is the volume of a QD, $P$ is Kane's parameter, ${ }^{33} I_{\text {overlap }}$ is the overlap integral of the electron and hole wave functions in a QD, $d$ is the degeneracy of the optical transition in a QD, $E(\lambda)=h c / \lambda$ is the incident photon energy, $\langle\ldots>$ means averaging over the inhomogeneously broadened ensemble of QDs, the deltafunction of energy in $\langle\ldots\rangle$ originates from the density of states in a $\mathrm{QD}, E_{0}=E_{\mathrm{g}}^{\mathrm{QD}}+\varepsilon_{\mathrm{n}}+\varepsilon_{\mathrm{p}}$ is the energy of the optical transition in a $\mathrm{QD}$, and $E_{\mathrm{g}}^{\mathrm{QD}}$ is the band-gap energy of the QD material.

The absorption coefficient $\alpha_{\mathrm{QD}}$ has a peak at the energy of the optical transition in a mean-sized QD $\bar{E}_{0}=E_{\mathrm{g}}^{\mathrm{QD}}$ $+\bar{\varepsilon}_{\mathrm{n}}+\bar{\varepsilon}_{\mathrm{p}}=h c / \bar{\lambda}_{0}$, where $\bar{\varepsilon}_{\mathrm{n}}$ and $\bar{\varepsilon}_{\mathrm{p}}$ are the electron and hole quantized energies in a mean-sized QD. Since $\alpha_{\mathrm{QD}}(\lambda)$ is very low for $\lambda$ far beyond $\bar{\lambda}_{0}$, we can safely use $+\infty$ as the upper limit for the integral in Eq. (27).
The current density associated with spontaneous radiative recombination of electrons and holes in QDs is

$$
j_{\mathrm{rec}}^{\mathrm{QD}}=q R_{\mathrm{QD}}=j_{\mathrm{rec}}^{\mathrm{QD} \_\mathrm{max}} f_{\mathrm{n}} f_{\mathrm{p}},
$$

where

$$
j_{\text {rec }}^{\mathrm{QD} \_\max }=q \frac{N_{\mathrm{S}}}{\tau_{\mathrm{QD}}}
$$

is the maximum value (when $f_{\mathrm{n}}=f_{\mathrm{p}}=1$ ) of $j_{\mathrm{rec}}^{\mathrm{QD}}, R_{\mathrm{QD}}$ is the radiative recombination rate in QDs

$$
R_{\mathrm{QD}}=N_{\mathrm{S}} \frac{f_{\mathrm{n}} f_{\mathrm{p}}}{\tau_{\mathrm{QD}}},
$$

and $\tau_{\mathrm{QD}}$ is the lifetime of spontaneous radiative recombination of an electron and a hole in a $\mathrm{QD}^{31,32}$

$$
\tau_{\mathrm{QD}}=\left[\frac{8}{3} \alpha_{\mathrm{fine}} \sqrt{\varepsilon_{\mathrm{r}}} \frac{\bar{E}_{0}}{\hbar}\left(\frac{P}{\hbar c}\right)^{2} I_{\text {overlap }}\right]^{-1} .
$$

The ratio of $j_{\mathrm{ph}}^{\mathrm{QD}}$ to $j_{\mathrm{rec}}^{\mathrm{QD}}$ is

$$
\frac{j_{\mathrm{ph}}^{\mathrm{QD}}}{j_{\mathrm{rec}}^{\mathrm{QD}}}=\frac{g_{\mathrm{QD}}}{R_{\mathrm{QD}}}=\frac{j_{\mathrm{ph}}^{\mathrm{QD}} \max }{j_{\mathrm{rec}}^{\mathrm{QD} \_\max }}\left\{\exp \left[\frac{\left(\bar{\varepsilon}_{\mathrm{n}}+\bar{\varepsilon}_{\mathrm{p}}\right)-\left(\mu_{\mathrm{n}}+\mu_{\mathrm{p}}\right)}{T}\right]-1\right\} .
$$

As seen from (34), to have $j_{\mathrm{ph}}^{\mathrm{QD}}$ positive, the condition $\bar{\varepsilon}_{\mathrm{n}}$ $+\bar{\varepsilon}_{\mathrm{p}}>\mu_{\mathrm{n}}+\mu_{\mathrm{p}}$ should hold. Note that the opposite of this inequality is the population inversion condition in a laser diode. This is reasonable since lasers are power-consumption devices and population inversion is achieved by injection of carriers, while a solar cell is a power source and hence there should be more carriers in lower energy states to have more absorption than recombination.

Using Eqs. (21), (25), (26), and (30), the total current density is obtained

$$
j=j_{\mathrm{ph}}-j_{\mathrm{s}}\left[\exp \left(\frac{V}{T}\right)-1\right]+j_{\mathrm{QD}},
$$

where

$$
\begin{gathered}
j_{\mathrm{ph}}=q \phi_{0} \frac{\beta_{2}^{2} e^{-\beta_{2}}}{\beta_{2}^{2}-\beta_{1}^{2}}\left(\frac{1+\beta_{1} \beta_{3} / \beta_{2}}{\beta_{3} \sinh \beta_{1}+\cosh \beta_{1}} e^{\beta_{2}}-\frac{\beta_{1}}{\beta_{2}} \frac{\beta_{3} \cosh \beta_{1}+\sinh \beta_{1}}{\beta_{3} \sinh \beta_{1}+\cosh \beta_{1}}-1\right) \\
+q \phi_{0} \frac{\beta_{6}^{2} e^{-\beta_{4}}}{\beta_{6}^{2}-\beta_{5}^{2}}\left(\frac{\beta_{5} \beta_{7} / \beta_{6}-1}{\beta_{7} \sinh \beta_{5}+\cosh \beta_{5}} e^{-\beta_{6}}-\frac{\beta_{5}}{\beta_{6}} \frac{\beta_{7} \cosh \beta_{5}+\sinh \beta_{5}}{\beta_{7} \sinh \beta_{5}+\cosh \beta_{5}}+1\right)+q \phi_{0}\left(e^{-\beta_{2}}-e^{-\beta_{4}}\right), \\
j_{\mathrm{s}}=\frac{q D_{\mathrm{p}} p_{\mathrm{no}}}{L_{\mathrm{p}}} \frac{\beta_{3} \cosh \beta_{1}+\sinh \beta_{1}}{\beta_{3} \sinh \beta_{1}+\cosh \beta_{1}}+\frac{q D_{\mathrm{n}} n_{\mathrm{po}}}{L_{\mathrm{n}}} \frac{\beta_{7} \cosh \beta_{5}+\sinh \beta_{5}}{\beta_{7} \sinh \beta_{5}+\cosh \beta_{5}}+q b B n_{\mathrm{i}}^{2} \\
j_{\mathrm{QD}}=j_{\mathrm{ph}}^{\mathrm{QD}}-j_{\mathrm{rec}}^{\mathrm{QD}},
\end{gathered}
$$

$L_{\mathrm{n}, \mathrm{p}}$ are the diffusion lengths for minority electrons and holes, respectively. Parameters $\beta_{1-7}$ (defined in Table I) are determined by the cell structure and optical properties of cell materials. ${ }^{25}$ 
With Eqs. (6) and (7), $j_{\mathrm{QD}}$ can also be written as

$$
\begin{aligned}
j_{\mathrm{QD}} & =q\left\{g_{\text {non_th, } \mathrm{n}}+\sigma_{\mathrm{n}} \mathrm{v}_{\mathrm{n}} N_{\mathrm{S}}\left(1-f_{\mathrm{n}}\right)\left[n_{1} \frac{f_{\mathrm{n}}}{1-f_{\mathrm{n}}}-\frac{n\left(x_{0}-0\right)+n\left(x_{0}+0\right)}{2}\right]\right\} \\
& =q\left\{g_{\text {non_th,p }}+\sigma_{\mathrm{p}} \mathrm{v}_{\mathrm{p}} N_{\mathrm{S}}\left(1-f_{\mathrm{p}}\right)\left[p_{1} \frac{f_{\mathrm{p}}}{1-f_{\mathrm{p}}}-\frac{p\left(x_{0}-0\right)+p\left(x_{0}+0\right)}{2}\right]\right\} .
\end{aligned}
$$

Since $j_{\mathrm{QD}}(V)$ can be voltage-dependent through such dependences of $f_{\mathrm{n}, \mathrm{p}}(V)$, for the short circuit current density and the open circuit voltage, we have

$$
\begin{gathered}
j_{\mathrm{sc}}=j_{\mathrm{ph}}+j_{\mathrm{QD}}(0), \\
V_{\mathrm{oc}}=T \ln \left[\frac{j_{\mathrm{ph}}+j_{\mathrm{QD}}\left(V_{\mathrm{oc}}\right)}{j_{\mathrm{s}}}+1\right] .
\end{gathered}
$$

It is worth noting that, if $j_{\mathrm{QD}}$ is voltage-dependent, the total current density cannot simply be written in the form of $j_{\mathrm{sc}}-j_{\mathrm{s}}[\exp (V / T)-1]$.

\section{Discussion}

There have been experimental reports showing that the position of the QD layer can play an important role in determining the cell performance. ${ }^{34,35}$ Due to the positiondependence of $f_{\mathrm{n}, \mathrm{p}}$ and $g_{\mathrm{QD}}$, the quantities $R_{\mathrm{QD}}$ and $j_{\mathrm{QD}}$ are also functions of position. Hence, our model can be used to study the effects of the QD layer position. We will, however, assume here that the QD layer is located in the middle of the i-region, which is the most common situation. We leave the effects of the QD layer position for a separate study.

Using our model, numerical calculations would allow us to obtain the spatial profiles of the electric field and carrier densities, as well as the energy band diagram. We are, however, able to derive here an analytical expression for the $j-V$ characteristic [Eq. (35)] without involving extensive numerical calculations.

The primary focus of our study is the factors limiting the QDSC performance. For this purpose, we will separately study the QDSC in the following three cases.

\section{Quasi-equilibrium between QDs and the bulk i-region}

If we assume that the carrier exchange between QDs and the i-region is instantaneous (i.e., the carrier capture

TABLE I. Expressions for parameters entering into Eqs. (36) and (37).

\begin{tabular}{ll}
\hline \hline Parameter & Expression \\
\hline$\beta_{1}$ & $w_{\mathrm{n}} / L_{\mathrm{p}}$ \\
$\beta_{2}$ & $w_{\mathrm{n}} \alpha_{\text {bulk }}$ \\
$\beta_{3}$ & $L_{\mathrm{p}} S_{\mathrm{p}} / D_{\mathrm{p}}$ \\
$\beta_{4}$ & $\left(b+w_{\mathrm{n}}\right) \alpha_{\text {bulk }}$ \\
$\beta_{5}$ & $w_{\mathrm{p}} / L_{\mathrm{n}}$ \\
$\beta_{6}$ & $w_{\mathrm{p}} \alpha_{\text {bulk }}$ \\
$\beta_{7}$ & $L_{\mathrm{n}} S_{\mathrm{n}} / D_{\mathrm{n}}$ \\
\hline \hline
\end{tabular}

cross-sections $\sigma_{\mathrm{n}, \mathrm{p}}=\infty$ and the tunneling escape of confined carriers to the i-region is instantaneous ${ }^{36}$ ), the quasiequilibrium will be established, the output voltage will be given as $V=E_{\mathrm{g}}^{\mathrm{QD}}+\mu_{\mathrm{n}}+\mu_{\mathrm{p}}$, and Eq. (34) can be rewritten as

$$
\frac{j_{\mathrm{ph}}^{\mathrm{QD}}}{j_{\mathrm{rec}}^{\mathrm{QD}}}=\frac{j_{\mathrm{ph}}^{\mathrm{QD} \_ \text {max }}}{j_{\mathrm{rec}}^{\mathrm{QD} \_ \text {max }}}\left[\exp \left(\frac{\bar{E}_{0}-V}{T}\right)-1\right] \text {. }
$$

Clearly, to have a positive $j_{\mathrm{ph}}^{\mathrm{QD}}$, the output voltage should be lower than $\bar{E}_{0} / q$.

Depending on the doping levels, conduction and valence band offsets, thickness of the i-region, QD mean size, and location of the QD layer, we will have the following two situations [(i) and (ii) below] in which expressions for $f_{\mathrm{n}, \mathrm{p}}$ can be obtained: ${ }^{29}$

(i) The QD layer is charge neutral. Then, we have

$$
f_{\mathrm{n}}=f_{\mathrm{p}}=f_{\mathrm{CN}}(V)=\left[1+\exp \left(\frac{\bar{E}_{0}-V}{2 T}\right)\right]^{-1} \text {. }
$$

Since $\bar{E}_{0} \gg T$, at short circuit $f_{\mathrm{CN}} \approx 0, j_{\mathrm{QD}}(0)$ $\approx j_{\mathrm{ph}}^{\mathrm{QD} \max }$, and $j_{\mathrm{sc}}$ becomes

$$
j_{\mathrm{sc}}=j_{\mathrm{ph}}+j_{\mathrm{ph}}^{\mathrm{QD} \_\mathrm{max}} .
$$

$V_{\mathrm{oc}}$ is calculated numerically from Eq. (41).

Fig. 2 shows $j_{\mathrm{ph}}^{\mathrm{QD}}, j_{\mathrm{rec}}^{\mathrm{QD}}$, and $j_{\mathrm{QD}}$ as functions of the output voltage. With increasing $V$, both $j_{\mathrm{ph}}^{\mathrm{QD}}$ and $j_{\mathrm{QD}}$ decrease (from $j_{\mathrm{ph}}^{\mathrm{QD} \max }=3.9 \mu \mathrm{A} / \mathrm{cm}^{2}$ ) and become zero as the voltage equals to 0.6 and $0.99 \mathrm{~V}$ [when $V=\bar{E}_{0}$-see Eq. (42)], respectively. $j_{\text {rec }}^{\mathrm{QD}}$ increases with $V$ and approaches $j_{\text {rec }}^{\text {QD_max }}(=32.8 \mathrm{~A} /$ $\mathrm{cm}^{2}$ ) as $V$ becomes much larger than $\bar{E}_{0}$. Since $j_{\text {rec }}^{\mathrm{QD} \_m a x} \gg j_{\mathrm{ph}}^{\mathrm{QD} \max }$, as $V$ approaches $\bar{E}_{0}, j_{\text {rec }}^{\mathrm{QD}}$ becomes larger than $j_{\mathrm{ph}}^{\mathrm{QD}}$ and thus $j_{\mathrm{QD}}$ becomes negative. In our calculations, the most commonly studied InAs/ GaAs QDSC is considered, and typical values for materials properties are used. ${ }^{37}$

Fig. 3 shows the $j$ - $V$ characteristic and the output electrical power densities $\left(\mathrm{mW} / \mathrm{cm}^{2}\right)$ for both the conventional SC and QDSC under different scenarios. The solid curves represent the conventional SC and the dashed-dotted curves represent the QDSC under quasi-equilibrium. The current density $j(V)$ for the conventional SC is calculated using Eq. (35) and neglecting the last term $j_{\text {QD }}$ therein. As seen from Eq. (44), $j_{\mathrm{sc}}$ for the QDSC is increased compared to that for the conventional SC. However, the increase is practically negligible. This is because of a very small 


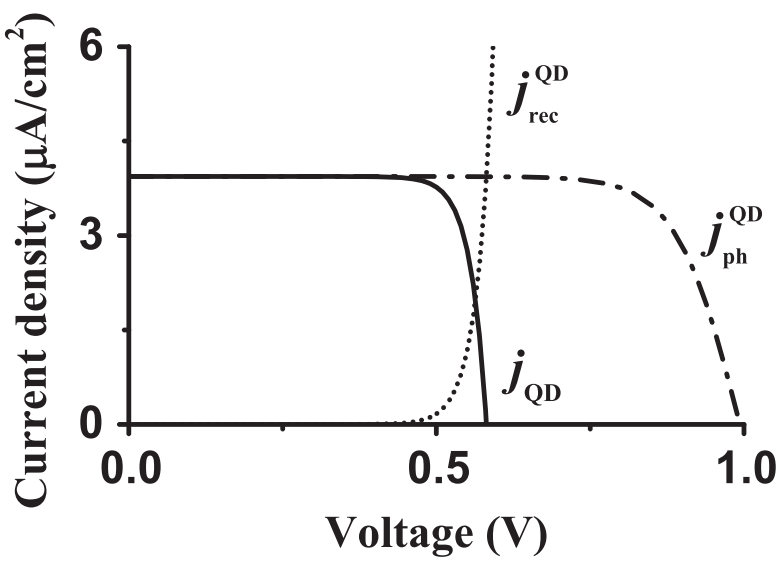

FIG. 2. Voltage dependences of current densities for the processes associated with QDs in a QDSC. The current densities due to photon absorption $\left(j_{\mathrm{ph}}^{\mathrm{QD}}\right)$, spontaneous radiative recombination $\left(j_{\text {rec }}^{\mathrm{QD}}\right)$, and $j_{\mathrm{QD}}=j_{\mathrm{ph}}^{\mathrm{QD}}-j_{\mathrm{rec}}^{\mathrm{QD}}$ are shown by the dashed-dotted, dotted, and solid curve, respectively. For the QDSC, the following two assumptions are made: (i) there is quasiequilibrium between QDs and the bulk i-region and (ii) the QD layer is charge neutral (i.e., $f_{\mathrm{n}}=f_{\mathrm{p}}$ ). At $0.58 \mathrm{~V}, j_{\mathrm{QD}}=0$, and at $\bar{E}_{0} / q=0.99 \mathrm{~V}, j_{\mathrm{ph}}^{\mathrm{QD}}$ $=0$ [see Eqs. (26) and (43)]. The mean size of QDs is $9 \mathrm{~nm}$, an infinitely deep potential well for confined carriers is assumed, the root mean square of QD-size fluctuations $\delta=0.05$, the overlap integral of the confined electron and hole wave functions $I_{\text {overlap }}=1$, the spontaneous radiative lifetime in a $\mathrm{QD} \tau_{\mathrm{QD}}=0.49 \mathrm{~ns}$, and the QD surface density (i.e., the number of QDs per unit area of the QD layer) $N_{\mathrm{S}}=10^{11} \mathrm{~cm}^{-2}$.

QD absorption coefficient as can be seen from Eq. (28). The major contribution to $j_{\mathrm{sc}}$ is still from the optical absorption by the bulk, not by QDs. On the other hand, $V_{\text {oc }}$ for the QDSC is much lower than that for the conventional SC. The reason for voltage degradation is in a very strong spontaneous radiative recombination in QDs. Since $f_{\mathrm{n}, \mathrm{p}}$ increase with $V, j_{\mathrm{ph}}^{\mathrm{QD}}$ decreases; however, $j_{\text {rec }}^{\mathrm{QD}}$ increases with $V$. Since $j_{\text {rec }}^{\mathrm{QD} \_\mathrm{max}} \gg j_{\mathrm{ph}}^{\mathrm{QD} \text { max }}, j_{\mathrm{QD}}$ is already negative at $V=V_{\mathrm{oc}}$ (see Fig. 2) and, therefore, the open circuit voltage for the QDSC is lower than its bulk counterpart. Our findings on a negligible increase in $j_{\mathrm{sc}}$ and a significant decrease in $V_{\text {oc }}$ in a QDSC are consistent with experimental reports. $3,7,8,19$

If multiple layers with QDs are used, it might seem that $j_{\mathrm{ph}}^{\mathrm{QD} \_m a x}$ could be multiplied by the number of QD layers $Z_{\mathrm{L}}$ to calculate $j_{\mathrm{sc}}$ and $V_{\mathrm{oc}}$. However, since $f_{\mathrm{n}, \mathrm{p}}$ depend on the position, each layer will now have its own values of $f_{\mathrm{n}, \mathrm{p}}$. Therefore, $j_{\mathrm{ph}}^{\mathrm{QD} \text { max }}$ should be calculated separately for each layer and the $j-V$ characteristic should be derived according to Eq. (20).

(ii) Charge neutrality is violated in the QD layer, i.e., $f_{\mathrm{n}} \neq f_{\mathrm{p}}$. Analytical expressions for $f_{\mathrm{n}, \mathrm{p}}$ for this situation have already been derived. ${ }^{29}$ It can be easily proved that, in this situation

$$
f_{\mathrm{n}} f_{\mathrm{p}} \leq f_{\mathrm{CN}}^{2}
$$

A smaller value of the product $f_{\mathrm{n}} f_{\mathrm{p}}$ means a weaker recombination in QDs [see Eq. (32)]. Hence, violation of charge neutrality in QDs is beneficial for better performance of QDSCs. Since the i-region of the QDSC structure considered here is considerably thicker than the QD layer, we did not take into account the effect

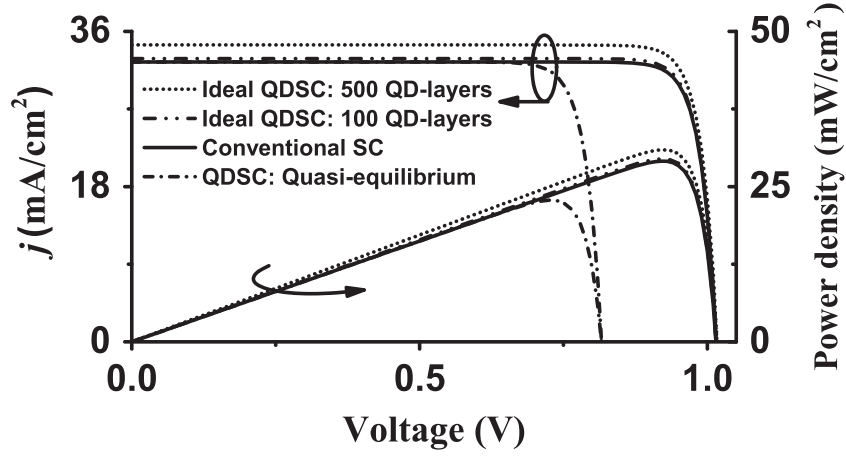

FIG. 3. Current density $j$ and output electrical power density as a function of the output voltage for the conventional SC (solid curves) and QDSC under different scenarios. The dashed-dotted curves for the QDSC are calculated assuming (i) quasi-equilibrium between QDs and the bulk and (ii) charge neutrality in the QD layer. The dotted and dashed-dotted-dotted curve represent the QDSC under the best-case scenario with the number of QD layers $Z_{\mathrm{L}}=500$ and 100 , respectively. $V_{\mathrm{oc}}$ for the QDSC under quasi-equilibrium is $0.81 \mathrm{~V}$, while $V_{\mathrm{oc}}=1.01 \mathrm{~V}$ is practically the same for the conventional SC and QDSCs under the best-case scenario. The power conversion efficiencies are $16.9 \%, 21.6 \%, 21.9 \%$, and $23.0 \%$ for the curves from bottom up, respectively.

of violation of charge neutrality in QDs. However, in QDSCs structures with a thinner i-region, the effect of violation of charge neutrality in QDs on the $j-V$ characteristic may be stronger.

\section{Best-case scenario: $f_{n}=f_{p}=0$ for the entire range of $V$}

In a QDSC, both photocarrier generation and recombination processes will occur in QDs. To have better performance, it is desirable to enhance the generation process while suppressing the carrier recombination process. This can be done by simultaneously decreasing $f_{\mathrm{n}}$ and $f_{\mathrm{p}}$ [see Eqs. (26) and (30)]. Ideally, we should have $j_{\mathrm{QD}}=j_{\mathrm{ph}}^{\mathrm{QD}}=j_{\mathrm{ph}}^{\mathrm{QD} \text { max }}$ and $j_{\text {rec }}^{\mathrm{QD}}=0$, i.e., $f_{\mathrm{n}}=f_{\mathrm{p}}=0$ for the entire range of $V$ (i.e., from 0 to $V_{\mathrm{oc}}$, the inequality $\bar{\varepsilon}_{\mathrm{n}, \mathrm{p}} \gg \mu_{\mathrm{n}, \mathrm{p}}$ is always satisfied). In such a case, there will only be photocarrier generation and no radiative recombination in QDs $\left(R_{\mathrm{QD}}=0\right)$; QDs will act as photocarrier generation centers and their inclusion into a conventional SC will lead to better performance-both the short circuit current density and open circuit voltage will be improved according to Eqs. (40) and (41), respectively. If $Z_{\mathrm{L}}$ layers with QDs are used and assuming the best-case scenario $\left(f_{\mathrm{n}}=f_{\mathrm{p}}=0\right)$ in each layer, $j_{\mathrm{sc}}$ and $V_{\mathrm{oc}}$ will be given by

$$
\begin{gathered}
j_{\mathrm{sc}}=j_{\mathrm{ph}}+Z_{\mathrm{L}} j_{\mathrm{ph}}^{\mathrm{QD} \_ \text {max }} \\
V_{\mathrm{oc}}=T \ln \left(\frac{j_{\mathrm{ph}}+Z_{\mathrm{L}} j_{\mathrm{ph} \_ \text {max }}^{\mathrm{QD}}}{j_{\mathrm{s}}}+1\right) .
\end{gathered}
$$

In this case, we obtain an analytical expression for the power conversion efficiency

$$
\eta_{\mathrm{best}}=\frac{V_{\mathrm{opt}}\left\{j_{\mathrm{sc}}-j_{\mathrm{s}}\left[\exp \left(V_{\mathrm{opt}} / T\right)-1\right]\right\}}{\int_{0}^{+\infty} F(\lambda) h c / \lambda d \lambda},
$$

where the optimum voltage $V_{\text {opt }}$, at which the output power density is maximum, is found from the solution of the equation 


$$
\left(1+\frac{V_{\mathrm{opt}}}{T}\right) \exp \left(\frac{V_{\mathrm{opt}}}{T}\right)=\frac{j_{\mathrm{sc}}+j_{\mathrm{s}}}{j_{\mathrm{s}}} .
$$

For this ideal case, the dotted and dashed-dotted-dotted curves in Fig. 3 are plotted for the QDSCs with 500 and 100 QD layers, respectively. Compared with the conventional SC (the solid curve) and non-ideal QDSC (the dashed-dotted curves), $j_{\mathrm{sc}}$ is increased due to absorption of sub-bandgap energy photons and elimination of radiative recombination at short circuit [see Eqs. (40) and (46)]. However, in view of a very small absorption coefficient [see Eq. (28)], the improvement in $j_{\mathrm{sc}}$ for the ideal QDSC with 100 QD layers is still quite small. Only with the use of large number of QD layers $\left(\mathrm{Z}_{\mathrm{L}}=500\right.$ for the top dotted curve), a large increase in $j_{\mathrm{sc}}$ is obtained. Compared with the non-ideal QDSC (the dasheddotted curve), a significant recovery in $V_{\mathrm{oc}}$ is obtained in the ideal QDSCs. The improvement in $V_{\mathrm{oc}}$ is due to elimination of strong spontaneous radiative recombination in QDs. Due to a logarithmic dependence on $j_{\mathrm{ph}}^{\mathrm{QD} \_ \text {max }}$ (with $j_{\mathrm{ph}}^{\mathrm{QD} \_ \text {max }}$ being very small) and $Z_{\mathrm{L}}$ [see Eq. (47)], $V_{\mathrm{oc}}$ for the ideal QDSC is practically the same as that for the conventional SC. This confirms again that it is the strong spontaneous radiative recombination of carriers in QDs, which is responsible for the open circuit voltage degradation in a QDSC.

In Fig. 3, the output electrical power densities (in units of $\mathrm{mW} / \mathrm{cm}^{2}$ ) are also shown. The power conversion efficiencies are $16.9 \%, 21.6 \%, 21.9 \%$, and $23.0 \%$ for the QDSC under quasi-equilibrium, conventional SC, and ideal QDSCs with $Z_{\mathrm{L}}=100$ and 500, respectively. As seen from the figure, a multi-layer QDSC will have a higher efficiency than its conventional counterpart if spontaneous radiative recombination of carriers in QDs is suppressed.

\section{Photoexcitation of confined carriers}

Carriers that are confined in QDs can absorb photons and thus be excited to continuum-energy states in the bulk. This process of photoexcitation is another possible mechanism for carriers to escape from QDs (process (5) in Fig. 1), especially under concentrated sunlight. The electron and hole photoexcitation coefficients (in units of $\mathrm{cm}^{-1}$ ) are given by $^{31,38}$

$$
\begin{aligned}
\beta_{\mathrm{ex}, \mathrm{n}, \mathrm{p}}(\lambda)= & 64 \sqrt{2} \pi^{3} \frac{1}{\bar{a}} \frac{\alpha_{\mathrm{finn}}}{\sqrt{\varepsilon_{\mathrm{r}}}} \frac{\Delta E_{\mathrm{c}, \mathrm{v}}}{h c / \lambda}\left(\frac{\hbar^{2}}{m_{\mathrm{c}, \mathrm{v}} \bar{a}^{2} h c / \lambda}\right)^{5 / 2} \\
& \times\left[f_{\mathrm{n}, \mathrm{p}}\left(\bar{\varepsilon}_{\mathrm{n}, \mathrm{p}}\right)-f_{\mathrm{n}, \mathrm{p}}\left(\bar{\varepsilon}_{\mathrm{n}, \mathrm{p}}+h c / \lambda\right)\right],
\end{aligned}
$$

where $m_{\mathrm{c}, \mathrm{v}}$ are the electron and hole effective masses in the QD material.

Depending on the mechanism by which photons are provided (incident sunlight or spontaneous radiative recombination in QDs), we will have the following two cases:

(i) If photons are provided by the incident sunlight, the photoexcitation rates are given as

$$
g_{\mathrm{ex}, \mathrm{n}, \mathrm{p}}=\int_{\lambda_{\text {bulk }}}^{\lambda_{\mathrm{c}, \mathrm{v}}} N_{\mathrm{S}} \sigma_{\mathrm{ex}, \mathrm{n}, \mathrm{p}} F(\lambda) d \lambda,
$$

where $\lambda_{\mathrm{c}, \mathrm{v}}$ are the longest wavelengths of photons, which correspond to the excitation energies $\Delta E_{\mathrm{c}, \mathrm{v}}-\bar{\varepsilon}_{\mathrm{n}, \mathrm{p}}$, and $\sigma_{\mathrm{ex}, \mathrm{n}, \mathrm{p}}(\lambda)=\bar{a}^{3} \beta_{\mathrm{ex}, \mathrm{n}, \mathrm{p}}(\lambda)$ are the cross-sections (in units of $\mathrm{cm}^{2}$ ) for the photoexcitation processes.

Fig. 4(a) shows the photoexcitation rates as functions of $f_{\mathrm{n}}\left(\bar{\varepsilon}_{\mathrm{n}}\right)$ at different values of the conduction band offset $\Delta E_{\mathrm{c}}$. If $f_{\mathrm{n}}=0$ or $1, g_{\mathrm{ex}, \mathrm{n}}=0$ since $\beta_{\mathrm{ex}, \mathrm{n}}$ becomes zero as is seen from Eq. (50). Depending on the values of $\Delta E_{\mathrm{c}}-\bar{\varepsilon}_{\mathrm{n}}$, there can be two different types for the dependence of $g_{\mathrm{ex}, \mathrm{n}}$ on $f_{\mathrm{n}}$. When $\Delta E_{\mathrm{c}}-\bar{\varepsilon}_{\mathrm{n}}$ is small, $g_{\mathrm{ex}, \mathrm{n}}$ increases with increasing $f_{\mathrm{n}}$, approaches its maximum value, and then decreases down to zero as $f_{\mathrm{n}}$ becomes unity. With increasing $\Delta E_{\mathrm{c}}$, the value of $f_{\mathrm{n}}$, at which $g_{\mathrm{ex}, \mathrm{n}}$ is maximum, increases and the maximum value of $g_{\mathrm{ex}, \mathrm{n}}$ decreases. When $\Delta E_{\mathrm{c}}-\bar{\varepsilon}_{\mathrm{n}}$ is large, $g_{\mathrm{ex}, \mathrm{n}}$ increases monotonically with $f_{\mathrm{n}}$ for $0 \leq f_{\mathrm{n}}<1$. The difference between the dependences is due to the following: when $\Delta E_{\mathrm{c}}-\bar{\varepsilon}_{\mathrm{n}}$ is small and comparable with $T$, as $f_{\mathrm{n}}\left(\bar{\varepsilon}_{\mathrm{n}}\right)$ approaches unity, $f_{\mathrm{n}}\left(\bar{\varepsilon}_{\mathrm{n}}+h c / \lambda_{\mathrm{c}}\right)$ becomes comparable with $f_{\mathrm{n}}\left(\bar{\varepsilon}_{\mathrm{n}}\right)$; however, when $\Delta E_{\mathrm{c}}-\bar{\varepsilon}_{\mathrm{n}}$ is much larger than $T, f_{\mathrm{n}}\left(\bar{\varepsilon}_{\mathrm{n}}\right)$ is always much larger than $f_{\mathrm{n}}\left(\bar{\varepsilon}_{\mathrm{n}}+h c / \lambda\right)$ for $0 \leq \lambda \leq \lambda_{\mathrm{c}}$.

Although $\beta_{\mathrm{ex}, \mathrm{n}} \propto \Delta E_{\mathrm{c}}$ [see (50)], a smaller $\Delta E_{\mathrm{c}}$ also means a longer $\lambda_{\mathrm{c}}$ in Eq. (51). Besides, since $\beta_{\mathrm{ex}, \mathrm{n}} /\left[f_{\mathrm{n}}\left(\bar{\varepsilon}_{\mathrm{n}}\right)\right.$ $\left.-f_{\mathrm{n}}\left(\bar{\varepsilon}_{\mathrm{n}}+h c / \lambda\right)\right]$ increases as $\lambda^{7 / 2}$ with increasing $\lambda$ [see (50)], absorption of longer wavelength photons is stronger. Therefore, the maximum value of $g_{\text {ex,n }}$ is achieved when $\Delta E_{\mathrm{c}}=\bar{\varepsilon}_{\mathrm{n}}$ (i.e., when $\lambda_{\mathrm{c}}$ is longest), as is shown by the top curve in Fig. 4(a).

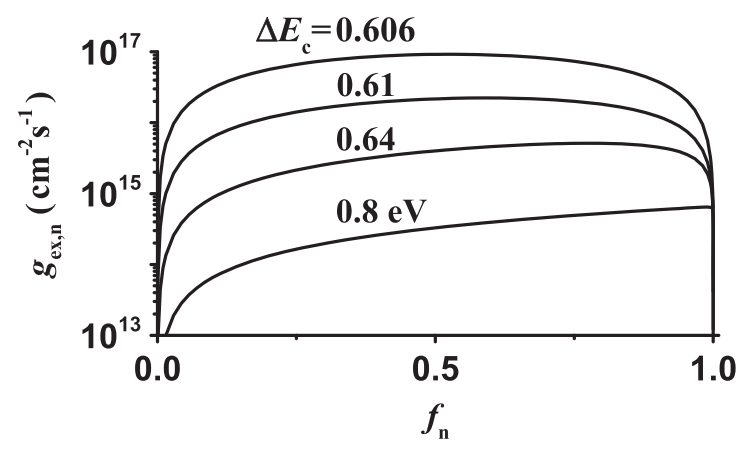

(a)

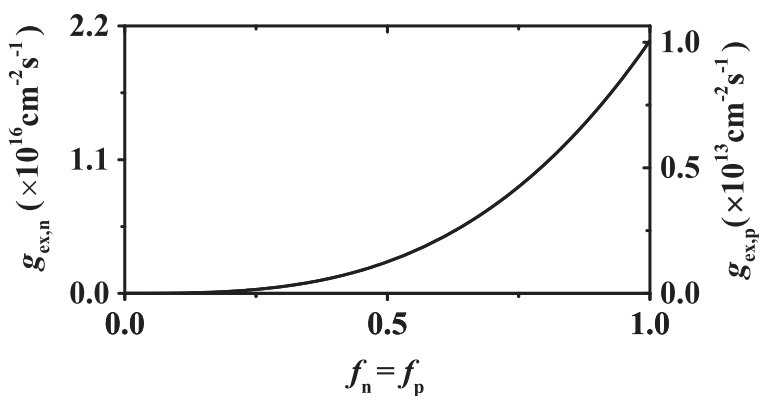

(b)

FIG. 4. Photoexcitation rate versus QD level occupancy for the cases when absorbed photons are provided by (a) the incident sunlight and (b) the spontaneous radiative recombination process in QDs. $\bar{\varepsilon}_{\mathrm{n}, \mathrm{p}}$ are 0.606 and $0.034 \mathrm{eV}$, respectively. In (a), the curves for $g_{\mathrm{ex}, \mathrm{n}}$ versus $f_{\mathrm{n}}$ are plotted at different conduction band offsets $\Delta E_{\mathrm{c}}$. In (b), $f_{\mathrm{n}}=f_{\mathrm{p}}$ is assumed and $\Delta E_{\mathrm{c}, \mathrm{v}}$ are 0.64 and $0.426 \mathrm{eV}$, respectively. 
The dependences of $g_{\mathrm{ex}, \mathrm{p}}$ on $f_{\mathrm{p}}$ are similar to those of $g_{\mathrm{ex}, \mathrm{n}}$ on $f_{\mathrm{n}}$. However, since the hole effective mass is much larger than the electron effective mass and since $\beta_{\text {ex,n,p }}$ are inversely proportional to $m_{\mathrm{c}, \mathrm{v}}^{5 / 2}, g_{\mathrm{ex}, \mathrm{p}}$ is several orders of magnitude smaller than $g_{\mathrm{ex}, \mathrm{n}}$.

(ii) If photons are provided by spontaneous radiative recombination in QDs, the expressions for $g_{\text {ex,n,p }}$ are

$$
g_{\mathrm{ex}, \mathrm{n}, \mathrm{p}}=N_{\mathrm{S}} \bar{a}^{3} \beta_{\mathrm{ex}, \mathrm{n}, \mathrm{p}}\left(\bar{\lambda}_{0}\right) R_{\mathrm{QD}} .
$$

Since the transition energy $h c / \bar{\lambda}_{0} \gg T$, then $f_{\mathrm{n}, \mathrm{p}}\left(\bar{\varepsilon}_{\mathrm{n}, \mathrm{p}}\right)$ $\gg f_{\mathrm{n}, \mathrm{p}}\left(\bar{\varepsilon}_{\mathrm{n}, \mathrm{p}}+h c / \bar{\lambda}_{0}\right)$ and $\beta_{\mathrm{ex}, \mathrm{n}, \mathrm{p}}\left(\bar{\lambda}_{0}\right) \propto f_{\mathrm{n}, \mathrm{p}}$. Hence, $g_{\mathrm{ex}, \mathrm{n}, \mathrm{p}} \propto f_{\mathrm{n}, \mathrm{p}}^{2} f_{\mathrm{p}, \mathrm{n}}$ depends on both $f_{\mathrm{n}}$ and $f_{\mathrm{p}}$. The ratio of the electron photoexcitation rate to that for holes is

$$
\frac{g_{\text {ex }, \mathrm{n}}}{g_{\text {ex }, \mathrm{p}}}=\frac{\Delta E_{\mathrm{c}}}{\Delta E_{\mathrm{v}}}\left(\frac{m_{\mathrm{v}}}{m_{\mathrm{c}}}\right)^{5 / 2} \frac{f_{\mathrm{n}}}{f_{\mathrm{p}}} .
$$

It is clear that when $f_{\mathrm{n}}=f_{\mathrm{p}}, g_{\mathrm{ex}, \mathrm{n}}$ will be much larger than $g_{\text {ex }, \mathrm{p}}$ since $m_{\mathrm{v}} \gg m_{\mathrm{c}}$.

Fig. 4(b) shows the dependences of $g_{\text {ex,n,p }}$ on the level occupancy for $f_{\mathrm{n}}=f_{\mathrm{p}}$. The maximum values (obtained when $f_{\mathrm{n}}=f_{\mathrm{p}}=1$ ) of the photoexcitation rates are $2.1 \times 10^{16}$ and $1.0 \times 10^{13} \mathrm{~cm}^{-2} \mathrm{~s}^{-1}$ for $g_{\text {ex,n, }, \text { }}$, respectively.

To study the effects of photoexcitation, let us assume that this process is the dominant mechanism for both electron and hole escape from QDs. Then from Eqs. (6) and (7), we have

$$
\begin{aligned}
& g_{\mathrm{ex}, \mathrm{n}}+R_{\mathrm{QD}}-g_{\mathrm{QD}}=0, \\
& g_{\mathrm{ex}, \mathrm{p}}+R_{\mathrm{QD}}-g_{\mathrm{QD}}=0 .
\end{aligned}
$$

As seen from (54) and (55), the photoexcitation rates are the same for electrons and holes

$$
g_{\mathrm{ex}, \mathrm{n}}=g_{\mathrm{ex}, \mathrm{p}} .
$$

If the net escape of confined carriers is due to the photoexcitation process, $j_{\mathrm{QD}}$ becomes

$$
j_{\mathrm{QD}}=q g_{\mathrm{ex}, \mathrm{n}}=q g_{\mathrm{ex}, \mathrm{p}} .
$$

For both cases discussed above, $f_{\mathrm{n}, \mathrm{p}}$ and thus $g_{\mathrm{ex}, \mathrm{n}, \mathrm{p}}$ and $j_{\mathrm{QD}}$ are found from the numerical solution of Eqs. (54) or (55). In contrast with the case of quasi-equilibrium, in which $f_{\mathrm{n}, \mathrm{p}}$ depend on the voltage [see Eq. (43)], $f_{\mathrm{n}, \mathrm{p}}$ for photoexcited carriers are voltage-independent. With Eq. (57), we immediately find from Eq. (41) that the open circuit voltage $V_{\mathrm{oc}}$ is larger than that for the conventional SC.

The maximum values of the photoexcitation rates are, however, several orders of magnitude smaller than the radiative recombination rate in QDs. For a typical InAs/GaAs QDSC considered here, the maximum values of $R_{\mathrm{QD}}$ (obtained when $f_{\mathrm{n}}=f_{\mathrm{p}}=1$ ), $g_{\mathrm{QD}}$ (obtained when $f_{\mathrm{n}}=f_{\mathrm{p}}=0$ ), and $g_{\text {ex,n }}$ [see Fig. 4(a)] are on the order of $10^{20}, 10^{13}$, and $10^{17} \mathrm{~cm}^{-2} \mathrm{~s}^{-1}$, respectively. The point is that, in order to satisfy Eqs. (54) and (55), $g_{\text {ex,n,p }}$ cannot be larger than $g_{\mathrm{QD}}$ and even if $g_{\mathrm{ex}, \mathrm{n}, \mathrm{p}}=g_{\mathrm{QD}}$, the maximum possible improvements in $j_{\mathrm{sc}}$ (on the order of $10^{-3} \mathrm{~mA} / \mathrm{cm}^{2}$ ) and $V_{\text {oc }}$ are negligible.
This means that practically no improvements in $j_{\mathrm{sc}}$ and $V_{\mathrm{oc}}$ will be brought about by the photoexcitation processes, and, as a result, the $j-V$ curve of the QDSC will be virtually the same as that of the SC without QDs (as shown by the solid curve in Fig. 3) if photoexcitation is the dominant mechanism for escape of confined carriers from QDs.

Hence, the fact of negligible improvement in the $j$-V characteristic is not only due to a very small photoexcitation coefficient but also to a large spontaneous radiative recombination rate and a very small optical absorption coefficient of QDs. To take advantages of photoexcitation, it is necessary (i) to increase the photon absorption by QDs and photoexcitation coefficients and (ii) to reduce the spontaneous radiative recombination rate $R_{\mathrm{QD}}$.

To reduce $R_{\mathrm{QD}}$, type-II energy band alignment between QDs and the host material can be used. In such structures, only one type of carriers is confined in QDs while the other is delocalized; as a result, electrons and holes are spatially separated so that the overlap integral of the electron and hole wave functions $I_{\text {overlap }}$ will be reduced, thus leading to an increase in the carrier lifetime [see Eq. (33)] and a reduction in $R_{\mathrm{QD}}$. There are both experimental and theoretical studies confirming that, due to such spatial separation of carriers, $\tau_{\mathrm{QD}}$ is increased from nano- to microsecond range. ${ }^{39,40}$ It should be noted that $I_{\text {overlap }}$ enters into both the photocarrier generation and radiative recombination rates. Reducing $I_{\text {overlap }}$ will reduce $R_{\mathrm{QD}}$; however, the $\mathrm{QD}$ absorption rate will also be reduced to the same extent [see Eq. (29)] —hence $I_{\text {overlap }}$ cancels in Eq. (34). Nevertheless, if concentrated sunlight is used, the reduction in absorption can be compensated and if, at the same time, $R_{\mathrm{QD}}$ remains reduced (in fact, while the absorption should ideally be proportional to the sunlight concentration [see Eq. (27)], $R_{\mathrm{QD}}$ has a maximum [see Eq. (31)]), the cell performance can be improved.

In this paper, the optical transitions only between the ground states of electrons and holes in QDs are considered. However, if the band offsets and/or QD sizes are large enough to support excited states, both $\beta_{\mathrm{ex}, \mathrm{n}, \mathrm{p}}$ and $\alpha_{\mathrm{QD}}$ will be increased, which will result in a stronger QD photon absorption and lead to better performance. Our theoretical model can be easily revised to include the presence of excited states in QDs—see Appendix B.

\section{CONCLUSIONS}

To conclude, we have developed a comprehensive theoretical model for semiconductor QD solar cells. In our model, the spatial discreteness of a QD layer is described by the Dirac delta-function and the carrier thermal exchange between QDs and the bulk i-region are characterized by the capture cross-sections. The coefficient of photon absorption (averaged over the inhomogeneously broadened ensemble of QDs) due to ground-state transitions in QDs is calculated as a function of the wavelength and QD level occupancy. The processes of carrier escape from QDs (namely, photoexcitation and tunneling) are also included in our model. The electron and hole current densities are shown to be discontinuous at the QD layer when the carrier generation and recombination rates in QDs are not equal to each other. A general 
analytical expression for the total current density in the QDSC is obtained and the current-voltage characteristic is studied for several specific situations. Our theoretical model confirms that, in line with earlier experimental studies, ${ }^{13}$ due to much stronger (compared with the photon absorption in QDs and photoexcitation from QDs) spontaneous radiative recombination in QDs, the performance of the solar cell can hardly be improved by using QDs no matter the dominant mechanism of escape of confined carriers from QDs to the bulk - thermal escape, tunneling, or photoexcitation.

(1) If QDs are at quasi-equilibrium with the bulk i-region, the expressions for the voltage-dependent $f_{\mathrm{n}, \mathrm{p}}$ can be obtained and the $j-V$ characteristic can be calculated. Compared with their conventional counterparts, $j_{\mathrm{sc}}$ is increased while $V_{\text {oc }}$ is decreased. Due to small absorption coefficient $\alpha_{\mathrm{QD}}$ of the QD ensemble, the improvement in $j_{\mathrm{sc}}$ is negligible. Strong spontaneous radiative recombination in QDs leads to large degradation in $V_{\mathrm{oc}}$.

(2) In the best-case scenario, when the spontaneous radiative recombination in QDs is completely suppressed (i.e., when $f_{\mathrm{n}}=f_{\mathrm{p}}=0$ ), both $j_{\mathrm{sc}}$ and the power conversion efficiency are higher than those in a conventional SC or in a non-ideal QDSC. Again, due to small value of $\alpha_{\mathrm{QD}}$, the increase in $j_{\mathrm{sc}}$ is negligible if only one QD layer is used. If multiple layers with QDs are used and the best-case scenario is maintained in each layer, $j_{\mathrm{sc}}$ will be much higher. $V_{\mathrm{oc}}$ in an ideal QDSC is much higher than that in a non-ideal QDSC. However, due to a logarithmic dependence on $Z_{\mathrm{L}}$ and $j_{\mathrm{ph}}^{\mathrm{QD} \_m a x}, V_{\mathrm{oc}}$ in an ideal QDSC is practically the same as that in the conventional SC.

(3) When photoexcitation is the dominant mechanism for escape of carriers from QDs to the bulk, $f_{\mathrm{n}, \mathrm{p}}$ become voltage-independent and both $j_{\mathrm{sc}}$ and $V_{\mathrm{oc}}$ are larger than those for the conventional SC. However, due to small values of $\alpha_{\mathrm{QD}}$ and $\beta_{\mathrm{ex}, \mathrm{n}, \mathrm{p}}$ and as well as a large value of $R_{\mathrm{QD}}$, the improvements in $j_{\mathrm{sc}}$ and $V_{\mathrm{oc}}$ are negligible-the maximum possible improvement in $j_{\mathrm{sc}}$ is on the order of $10^{-3} \mathrm{~mA} / \mathrm{cm}^{2}$. As a result, the $j-V$ curve is practically the same as that for the conventional SC.

\section{ACKNOWLEDGMENTS}

Y.W. acknowledges the support of the Fundamental Research Funds for the Central Universities (Grant No. 2015QNA10). L.V.A. acknowledges the U.S. Army Research Office (Grant No. W911NF-13-1-0445) for support of this work.

\section{APPENDIX A: TUNNELING BETWEEN NEIGHBORING QD LAYERS}

If multiple layers with QDs are used, tunneling can occur between neighboring QD layers. As shown in Fig. 5, carriers confined in a given QD layer can tunnel out to the neighboring layers located on its left- and/or right-hand sides. Carriers can also tunnel into a given QD layer from its neighboring layers. Hence, the rate equations for carriers confined in each QD layer should be revised to include the in- and out-tunneling terms. Denote the number of QD layers as $Z_{\mathrm{L}}$. We have then the following rate equations:

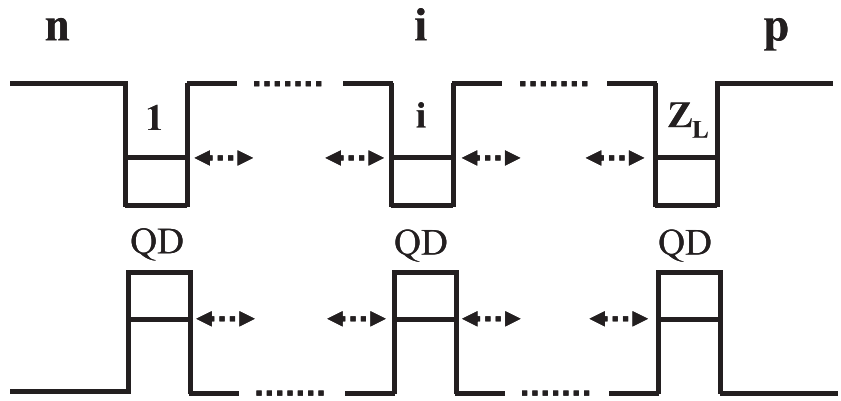

FIG. 5. Tunneling (shown by arrows) between neighboring QD layers in a QDSC. Out- and in-tunneling of carriers occur between a QD layer and its neighboring layers on the left- and/or right-hand sides.

For electrons confined in the 1st QD layer (counted from the left)

$$
\begin{aligned}
& \sigma_{\mathrm{n}} \mathrm{v}_{\mathrm{n}} N_{\mathrm{S}}\left(1-f_{\mathrm{n}, 1}\right) n-\sigma_{\mathrm{n}} \mathrm{v}_{\mathrm{n}} n_{1} N_{\mathrm{S}} f_{\mathrm{n}, 1} \\
& \quad+g_{\mathrm{QD}, 1}-R_{\mathrm{QD}, 1}-g_{\text {non_th }, 1}+R_{\mathrm{in}-\text { tunn }, 1}^{\mathrm{R}}-R_{\mathrm{out}-\text { tunn }, 1}^{\mathrm{R}}=0
\end{aligned}
$$

for electrons confined in the $\mathrm{i}$-th layer $\left(2 \leq \mathrm{i} \leq Z_{\mathrm{L}}-1\right)$

$$
\begin{aligned}
& \sigma_{\mathrm{n}} \mathrm{V}_{\mathrm{n}} N_{\mathrm{S}}\left(1-f_{\mathrm{n}, \mathrm{i}}\right) n-\sigma_{\mathrm{n}} \mathrm{V}_{\mathrm{n}} n_{1} N_{\mathrm{S}} f_{\mathrm{n}, \mathrm{i}}+g_{\mathrm{QD}, \mathrm{i}}-R_{\mathrm{QD}, \mathrm{i}}-g_{\text {non_th,i }} \\
& \quad+R_{\mathrm{in}-\text { tunn, } \mathrm{i}}^{\mathrm{R}}-R_{\text {out-tunn, } \mathrm{i}}^{\mathrm{R}}+R_{\mathrm{in}-\text { tunn,i }}^{\mathrm{L}}-R_{\text {out-tunn,i }}^{\mathrm{L}}=0,
\end{aligned}
$$

and for electrons confined in the $Z_{\mathrm{L}}$-th layer

$$
\begin{aligned}
& \sigma_{\mathrm{n}} \mathrm{V}_{\mathrm{n}} N_{\mathrm{S}}\left(1-f_{\mathrm{n}, \mathrm{Z}_{\mathrm{L}}}\right) n-\sigma_{\mathrm{n}} \mathrm{v}_{\mathrm{n}} n_{1} N_{\mathrm{S}} f_{\mathrm{n}, \mathrm{Z}_{\mathrm{L}}}+g_{\mathrm{QD}, \mathrm{Z}_{\mathrm{L}}} \\
& \quad-R_{\mathrm{QD}, \mathrm{Z}_{\mathrm{L}}}-g_{\text {non_th, }, \mathrm{Z}_{\mathrm{L}}}+R_{\mathrm{in} \text {-tunn, }, \mathrm{Z}_{\mathrm{L}}}^{\mathrm{L}}-R_{\text {out-tunn, }, \mathrm{Z}_{\mathrm{L}}}^{\mathrm{L}}=0,
\end{aligned}
$$

where $R_{\text {in-tunn }}$ and $R_{\text {out-tunn }}$ are the in- and out-tunneling rates of confined electrons, respectively. Superscripts "L" and "R" denote the tunneling exchange rates between a given QD layer and its left- and right-hand side neighbor, respectively. For the sake of simplicity, the Dirac delta-function $\delta\left(x-x_{\mathrm{i}}\right)$ $\left(x_{\mathrm{i}}\right.$ is the location of the $\mathrm{i}$-th layer) is dropped in each term in Eqs. (A1)-(A3). Similar equations can also be written for the confined holes.

As is clear from Eqs. (A1)-(A3), the following relations hold:

$$
\begin{aligned}
& R_{\text {in-tunn }, \mathrm{i}}^{\mathrm{R}}=R_{\text {out-tunn }, \mathrm{i}+1}^{\mathrm{L}}, \\
& R_{\text {out-tunn }, \mathrm{i}}^{\mathrm{R}}=R_{\text {in-tunn, }, \mathrm{i}+1}^{\mathrm{L}} .
\end{aligned}
$$

Similarly to Eq. (12), we will have the following boundary conditions at the layers:

$$
\begin{aligned}
\left.j_{\mathrm{n}}\right|_{x=x_{1}+0}-\left.j_{\mathrm{n}}\right|_{x=x_{1}-0} & \\
=q\left(R_{\mathrm{QD}, 1}-\right. & \left.g_{\mathrm{QD}, 1}+R_{\text {out-tunn }, 1}^{\mathrm{R}}-R_{\mathrm{in}-\text { tunn }, 1}^{\mathrm{R}}\right), \\
\left.j_{\mathrm{n}}\right|_{x=x_{\mathrm{i}}+0}-\left.j_{\mathrm{n}}\right|_{x=x_{\mathrm{i}}-0}= & q\left(R_{\mathrm{QD}, \mathrm{i}}-g_{\mathrm{QD}, \mathrm{i}}+R_{\text {out-tunn, } \mathrm{i}}^{\mathrm{R}}\right. \\
& \left.-R_{\text {in-tunn,i } \mathrm{i}}^{\mathrm{R}}+R_{\text {out-tunn }, \mathrm{i}}^{\mathrm{L}}-R_{\text {in }- \text { tunn, } \mathrm{i}}^{\mathrm{L}}\right),
\end{aligned}
$$




$$
\begin{aligned}
\left.j_{\mathrm{n}}\right|_{x=x_{\mathrm{Z}_{\mathrm{L}}}+0}-\left.j_{\mathrm{n}}\right|_{x=x_{\mathrm{Z}_{\mathrm{L}}}-0} \\
\quad=q\left(R_{\mathrm{QD}, \mathrm{Z}_{\mathrm{L}}}-g_{\mathrm{QD}, \mathrm{Z}_{\mathrm{L}}}+R_{\text {out-tunn, }, \mathrm{Z}_{\mathrm{L}}}^{\mathrm{L}}-R_{\text {in-tunn, }, \mathrm{Z}_{\mathrm{L}}}^{\mathrm{L}}\right) .
\end{aligned}
$$

Integrating Eq. (10) and using Eqs. (A4)-(A8), we obtain for the total current density

$$
j=j_{\mathrm{p}}(0)+q \int\left(g_{\text {bulk }}-R_{\text {bulk }}\right) d x+q \sum_{\mathrm{i}=1}^{\mathrm{Z}_{\mathrm{L}}}\left(g_{\mathrm{QD}, \mathrm{i}}-R_{\mathrm{QD}, \mathrm{i}}\right)+j_{\mathrm{n}}(b) .
$$

Although tunneling affects the $j-V$ characteristic through $f_{\mathrm{n}, \mathrm{p}}$, Eq. (A9) has exactly the same form as that for the case of no tunneling between neighboring QD layers [Eq. (20)].

\section{APPENDIX B: QDSC IN THE PRESENCE OF EXCITED STATES IN QDS}

Consider now a situation when excited states (ES) are present in QDs. Let us assume that the carrier exchange between QDs and the i-region is ES-mediated only. At the steady-state, we can write the following rate equations for electrons:

For excited state electrons

$$
\begin{aligned}
0= & \sigma_{\mathrm{n}} \mathrm{v}_{\mathrm{n}} N_{\mathrm{S}}\left(1-f_{\mathrm{n} 2}\right) n(x) \delta\left(x-x_{0}\right)-\sigma_{\mathrm{n}} \mathrm{v}_{\mathrm{n}} N_{\mathrm{S}} f_{\mathrm{n} 2} n_{2} \delta\left(x-x_{0}\right) \\
& +g_{\mathrm{QD} 2} \delta\left(x-x_{0}\right)-R_{\mathrm{QD} 2} \delta\left(x-x_{0}\right)+g_{\mathrm{QD}, 1 \rightarrow 2} \delta\left(x-x_{0}\right) \\
& +N_{\mathrm{S}} \frac{f_{\mathrm{n} 1}\left(1-f_{\mathrm{n} 2}\right)}{\tau_{12}} \delta\left(x-x_{0}\right)-N_{\mathrm{S}} \frac{f_{\mathrm{n} 2}\left(1-f_{\mathrm{n} 1}\right)}{\tau_{21}} \delta\left(x-x_{0}\right)
\end{aligned}
$$

for ground-state (GS) electrons

$$
\begin{aligned}
0= & g_{\mathrm{QD} 1} \delta\left(x-x_{0}\right)-R_{\mathrm{QD} 1} \delta\left(x-x_{0}\right)-g_{\mathrm{QD}, 1 \rightarrow 2} \delta\left(x-x_{0}\right) \\
& -N_{\mathrm{S}} \frac{f_{\mathrm{n} 1}\left(1-f_{\mathrm{n} 2}\right)}{\tau_{12}} \delta\left(x-x_{0}\right)+N_{\mathrm{S}} \frac{f_{\mathrm{n} 2}\left(1-f_{\mathrm{n} 1}\right)}{\tau_{21}} \delta\left(x-x_{0}\right),
\end{aligned}
$$

and for free electrons in the i-region

$$
\begin{aligned}
0= & \sigma_{\mathrm{n}} \mathrm{v}_{\mathrm{n}} n_{2} N_{\mathrm{S}} f_{\mathrm{n} 2} \delta\left(x-x_{0}\right)-\sigma_{\mathrm{n}} \mathrm{v}_{\mathrm{n}} N_{\mathrm{S}}\left(1-f_{\mathrm{n} 2}\right) n(x) \delta\left(x-x_{0}\right) \\
& -R_{\text {bulk }}+g_{\text {bulk }}+\frac{1}{q} \frac{d j_{\mathrm{n}}}{d x} .
\end{aligned}
$$

Similar equations can also be written for holes. "1" and " 2 " in the subscripts represent the GS and ES, respectively. $g_{\mathrm{OD}, 1->2}$ is the transition rate of carriers from ground to excited state due to the absorption of photons. $\tau_{12}$ is the ground-to-excited state transition time, and $\tau_{21}$ is the excited-to-ground state relaxation time in a QD.

Integrating Eq. (B3) over an infinitesimal interval around $x_{0}$ and using Eqs. (B1) and (B2), we have

$$
\begin{aligned}
& \frac{1}{q}\left(\left.j_{\mathrm{n}}\right|_{x=x_{0}+0}-\left.j_{\mathrm{n}}\right|_{x=x_{0}-0}\right) \\
& \quad=\sigma_{\mathrm{n}} \mathrm{v}_{\mathrm{n}} N_{\mathrm{S}}\left(1-f_{\mathrm{n} 2}\right) \frac{n\left(x_{0}+0\right)+n\left(x_{0}-0\right)}{2}-\sigma_{\mathrm{n}} \mathrm{v}_{\mathrm{n}} n_{2} N_{\mathrm{S}} f_{\mathrm{n} 2} \\
& \quad=\left(R_{\mathrm{QD} 1}+R_{\mathrm{QD} 2}\right)-\left(g_{\mathrm{QD} 1}+g_{\mathrm{QD} 2}\right) .
\end{aligned}
$$

The expression for the total current density will then be

$$
\begin{aligned}
j= & q \int\left(g_{\text {bulk }}-R_{\text {bulk }}\right) d x+q\left(g_{\mathrm{QD} 1}+g_{\mathrm{QD} 2}\right) \\
& -q\left(R_{\mathrm{QD} 1}+R_{\mathrm{QD} 2}\right)
\end{aligned}
$$

which is similar to Eq. (16). The total current density is again due to the net photocarrier generation by the entire device.

${ }^{1}$ A. Martí, L. Cuadra, and A. Luque, "Quantum dot intermediate band solar cell," in Proceesings of the 28th IEEE Photovoltaic Specialists Conference (PVSC) (2000), pp. 940-943.

${ }^{2}$ A. J. Nozik, "Quantum dot solar cells," Physica E 14(1-2), 115-120 (2002).

${ }^{3}$ A. Luque, A. Martí, C. Stanley, N. López, L. Cuadra, D. Zhou, J. L. Pearson, and A. McKee, "General equivalent circuit for intermediate band devices: Potentials, currents and electroluminescence," J. Appl. Phys. 96(1), 903-909 (2004).

${ }^{4}$ C. G. Bailey, D. V. Forbes, S. J. Polly, Z. S. Bittner, Y. S. Dai, C. Mackos, R. P. Raffaelle, and S. M. Hubbard, "Open-circuit voltage improvement of InAs/GaAs quantum-dot solar cells using reduced InAs coverage," IEEE J. Photovoltaics 2(3), 269-275 (2012).

${ }^{5}$ S. A. Blokhin, A. V. Sakharov, A. M. Nadtochy, A. S. Pauysov, M. V. Maximov, N. N. Ledentsov, A. R. Kovsh, S. S. Mikhrin, V. M. Lantratov, S. A. Mintairov, N. A. Kaluzhniy, and M. Z. Shvarts, "AlGaAs/GaAs photovoltaic cells with an array of InGaAs QDs," Semiconductors 43(4), 514-518 (2009).

${ }^{6}$ S. M. Hubbard, C. Bailey, S. Polly, C. Cress, J. Andersen, D. Forbes, and R. P. Raffaelle, "Nanostructured photovoltaics for space power," J. Nanophotonics 3(1), 031880 (2009).

${ }^{7}$ D. Guimard, R. Morihara, D. Bordel, K. Tanabe, Y. Wakayama, M. Nishioka, and Y. Arakawa, "Fabrication of InAs/GaAs quantum dot solar cells with enhanced photocurrent and without degradation of open circuit voltage," Appl. Phys. Lett. 96(20), 203507 (2010).

${ }^{8}$ K. A. Sablon, J. W. Little, V. Mitin, A. Sergeev, N. Vagidov, and K. Reinhardt, "Strong enhancement of solar cell efficiency due to quantum dots with built-in charge," Nano Lett. 11(6), 2311-2317 (2011).

${ }^{9} \mathrm{~V}$. Popescu and A. Zunger, "Three-dimensional assemblies of semiconductor quantum dots in a wide-gap matrix providing an intermediate band for absorption," J. Appl. Phys. 112(11), 114320 (2012).

${ }^{10}$ T. Sugaya, O. Numakami, R. Oshima, S. Furue, H. Komaki, T. Amano, K. Matsubara, Y. Okano, and S. Niki, "Ultra-high stacks of InGaAs/GaAs quantum dots for high efficiency solar cells," Energy Environ. Sci. 5(3), 6233-6237 (2012).

${ }^{11}$ F. K. Tutu, P. Lam, J. Wu, N. Miyashita, Y. Okada, K. H. Lee, N. J. Ekins-Daukes, J. Wilson, and H. Liu, "InAs/GaAs quantum dot solar cell with an AlAs cap layer," Appl. Phys. Lett. 102(16), 163907 (2013).

${ }^{12}$ G. Jolley, L. Fu, H. F. Lu, H. H. Tan, and C. Jagadish, "The role of intersubband optical transitions on the electrical properties of InGaAs/GaAs quantum dot solar cells," Prog. Photovoltaics: Res. Appl. 21(4), 736-746 (2013).

${ }^{13}$ D. G. Sellers, S. J. Polly, Y. J. Zhong, S. M. Hubbard, J. M. O. Zide, and M. F. Doty, "New nanostructured materials for efficient photon upconversion," IEEE J. Photovoltaics 5(1), 224-228 (2015).

${ }^{14} \mathrm{M}$. Wolf, "Limitations and possibilities for improvement of photovoltaic solar energy converters: Part I: Considerations for earth's surface operation," Proc. IRE 48(7), 1246-1263 (1960).

${ }^{15}$ A. Luque and A. Martí, "Increasing the efficiency of ideal solar cells by photon induced transitions at intermediate levels," Phys. Rev. Lett. 78(26), 5014-5017 (1997)

${ }^{16} \mathrm{~W}$. Shockley and H. J. Queisser, "Detailed balance limit of efficiency of pn junction solar cells," J. Appl. Phys. 32(3), 510-519 (1961).

${ }^{17}$ V. Aroutiounian, S. Petrosyan, A. Khachatryan, and K. Touryan, "Quantum dot solar cells," J. Appl. Phys. 89(4), 2268-2271 (2001).

${ }^{18}$ A. Martí, L. Cuadra, and A. Luque, "Quasi-drift diffusion model for the quantum dot intermediate band solar cell," IEEE Trans. Electron Devices 49(9), 1632-1639 (2002).

${ }^{19}$ A. Luque, A. Martí, N. López, E. Antolín, E. Cánovas, C. Stanley, C. Farmer, and P. Díaz, "Operation of the intermediate band solar cell under nonideal space charge region conditions and half filling of the intermediate band," J. Appl. Phys. 99(9), 094503 (2006).

${ }^{20}$ A. S. Lin and J. D. Phillips, "Drift-diffusion modeling for impurity photovoltaic devices," IEEE Trans. Electron Devices 56(12), 3168-3174 (2009). 
${ }^{21}$ R. Strandberg and T. W. Reenaas, "Photofilling of intermediate bands," J. Appl. Phys. 105(12), 124512 (2009).

${ }^{22}$ K. Yoshida, Y. Okada, and N. Sano, "Self-consistent simulation of intermediate band solar cells: Effect of occupation rates on device characteristics," Appl. Phys. Lett. 97(13), 133503 (2010).

${ }^{23}$ L. V. Asryan, S. Luryi, and R. A. Suris, "Internal efficiency of semiconductor lasers with a quantum-confined active region," IEEE J. Quantum Electron. 39(3), 404-418 (2003).

${ }^{24}$ L. V. Asryan and R. A. Suris, "Longitudinal spatial hole burning in a quantum-dot laser," IEEE J. Quantum Electron. 36(10), 1151-1160 (2000).

${ }^{25}$ S. J. Fonash, Solar Cell Device Physics, 2nd ed. (Academic Press/Elsevier, Burlington, MA, 2010).

${ }^{26}$ E. O. Kane, "Band structure of indium antimonide," J. Phys. Chem. Solids 1(4), 249-261 (1957).

${ }^{27}$ A. Luque and S. Hegedus, Handbook of Photovoltaic Science and Engineering, 2nd ed. (Wiley, Hoboken, NJ, 2011).

${ }^{28}$ S. M. Sze and K. K. Ng, Physics of Semiconductor Devices, 3rd ed. (Wiley-Interscience, Hoboken, NJ, 2007).

${ }^{29}$ L. V. Asryan and R. A. Suris, "Charge neutrality violation in quantum-dot lasers," IEEE J. Sel. Top. Quantum Electron. 3(2), 148-157 (1997).

${ }^{30}$ C. T. Sah, R. N. Noyce, and W. Shockley, "Carrier generation and recombination in $p-n$ junctions and $p-n$ junction characteristics," Proc. IRE 45(9), 1228-1243 (1957).

${ }^{31}$ L. V. Asryan and R. A. Suris, "Inhomogeneous line broadening and the threshold current density of a semiconductor quantum dot laser," Semicond. Sci. Technol. 11(4), 554-567 (1996).
${ }^{32}$ L. V. Asryan, M. Grundmann, N. N. Ledentsov, O. Stier, R. A. Suris, and D. Bimberg, "Maximum modal gain of a self-assembled InAs/GaAs quantum-dot laser,” J. Appl. Phys. 90(3), 1666-1668 (2001).

${ }^{33}$ L. A. Coldren, S. W. Corzine, and M. L. Mašanović, Diode Lasers and Photonic Integrated Circuits, 2nd ed. (Wiley, Hoboken, NJ, 2012).

${ }^{34}$ D. Zhou, P. E. Vullum, G. Sharma, S. F. Thomassen, R. Holmestad, T. W. Reenaas, and B. O. Fimland, "Positioning effects on quantum dot solar cells grown by molecular beam epitaxy," Appl. Phys. Lett. 96(8), 083108 (2010).

${ }^{35}$ K. Driscoll, M. F. Bennett, S. J. Polly, D. V. Forbes, and S. M. Hubbard, "Effect of quantum dot position and background doping on the performance of quantum dot enhanced GaAs solar cells," Appl. Phys. Lett. 104(2), 023119 (2014).

${ }^{36}$ D. S. Han and L. V. Asryan, "Output power of a double tunnelinginjection quantum dot laser," Nanotechnology 21(1), 015201 (2010).

${ }^{37}$ M. Shur, Physics of Semiconductor Devices (Prentice Hall, Upper Saddle River, NJ, 1990).

${ }^{38}$ L. V. Asryan and R. A. Suris, "Carrier photoexcitation from levels in quantum dots to states of the continuum in lasing," Semiconductors 35(3), 343-346 (2001).

${ }^{39}$ K. Nishikawa, Y. Takeda, T. Motohiro, D. Sato, J. Ota, N. Miyashita, and Y. Okada, "Extremely long carrier lifetime over 200 ns in GaAs wallinserted type II InAs quantum dots," Appl. Phys. Lett. 100(11), 113105 (2012).

${ }^{40} \mathrm{~S}$. Tomić, "Effect of Sb induced type II alignment on dynamical processes in InAs/GaAs/GaAsSb quantum dots: Implication to solar cell design," Appl. Phys. Lett. 103(7), 072112 (2013). 